\title{
Magnetization reversal of in-plane uniaxial Co films and its dependence on epitaxial alignment
}

\author{
O. Idigoras, ${ }^{1, a)}$ A. K. Suszka, ${ }^{1, b)}$ P. Vavassori, ${ }^{1,2}$ B. Obry, ${ }^{3}$ B. Hillebrands, ${ }^{3}$ P. Landeros, ${ }^{4}$ \\ and A. Berger ${ }^{1}$ \\ ${ }^{1}$ CIC nanoGUNE Consolider, Tolosa Hiribidea 76, E-20018 Donostia-San Sebastian, Spain \\ ${ }^{2}$ IKERBASQUE, The Basque Foundation for Science, E-48011 Bilbao, Spain \\ ${ }^{3}$ Fachbereich Physik and Landesforschungzentrum OPTIMAS, Technische Universität Kaiserslautern, \\ Erwin-Schrödinger-Straße 56, D-67663 Kaiserslautern, Germany \\ ${ }^{4}$ Departamento de Física, Universidad Técnica Federico Santa María, Avenida España 1680, \\ 2390123 Valparaíso, Chile
}

(Received 15 November 2013; accepted 14 February 2014; published online 28 February 2014)

\begin{abstract}
This work studies the influence of crystallographic alignment onto magnetization reversal in partially epitaxial Co films. A reproducible growth sequence was devised that allows for the continuous tuning of grain orientation disorder in Co films with uniaxial in-plane anisotropy by the controlled partial suppression of epitaxy. While all stable or meta-stable magnetization states occurring during a magnetic field cycle exhibit a uniform magnetization for fully epitaxial samples, non-uniform states appear for samples with sufficiently high grain orientation disorder. Simultaneously with the occurrence of stable domain states during the magnetization reversal, we observe a qualitative change of the applied field angle dependence of the coercive field. Upon increasing the grain orientation disorder, we observe a disappearance of transient domain wall propagation as the dominating reversal process, which is characterized by an increase of the coercive field for applied field angles away from the easy axis for well-ordered epitaxial samples. Upon reaching a certain disorder threshold level, we also find an anomalous magnetization reversal, which is characterized by a non-monotonic behavior of the remanent magnetization and coercive field as a function of the applied field angle in the vicinity of the nominal hard axis. This anomaly is a collective reversal mode that is caused by disorder-induced frustration and it can be qualitatively and even quantitatively explained by means of a two Stoner-Wohlfarth particle model. Its predictions are furthermore corroborated by Kerr microscopy and by Brillouin light scattering measurements. (C) 2014 AIP Publishing LLC. [http://dx.doi.org/10.1063/1.4867001]
\end{abstract}

\section{INTRODUCTION}

Magnetization reversal of thin films has been an active research area during the past decades, both in theoretical ${ }^{1-12}$ and experimental ${ }^{4,13-57}$ studies. Hereby, a large number of experimental works have been performed using ultra-thin films of only a few atomic layer thickness ${ }^{4,18,25,29,43}$ or multilayers, ${ }^{37,38,41,47}$ including magnetically coupled multilayers, for which interesting and relevant collective phenomena occur. ${ }^{29}$ For instance, the antiferromagnetic coupling of two ferromagnetic thin films across a nonferromagnetic metallic layer can result in strongly field-dependent magnetic states, which also leads to the well-known giant-magnetoresistance ${ }^{58-60}$ effect. Other examples are the exchange bias phenomenon as the result of an interface interaction in between antiferromagnetic and ferromagnetic layers ${ }^{23,31,56}$ and the exchange spring effect, which is the collective behavior of interface-coupled hard and soft ferromagnetic thin films. ${ }^{21,46,61}$ On the other hand, many studies have been performed for the purpose of analyzing the magnetization reversal mechanism in individual ferromagnetic films, including

\footnotetext{
a) o.idigoras@nanogune.eu

${ }^{b}$ Present address: ETH Zurich, Paul Scherrer Institute 5232 Villigen PSI, Switzerland.
}

magnetic alloy films. ${ }^{20,22,26,28,36,42,44,45,49,51,52,55,57}$ Hereby, many works have been focused on alloys made from $4 \mathrm{~d} / 5 \mathrm{~d}$ transition metals and $3 \mathrm{~d}$ elemental ferromagnets ${ }^{14,26,42,49}$ or alloys of $4 \mathrm{f}$ elements with $3 \mathrm{~d}$ elemental ferromagnets. ${ }^{17,22,55}$ These types of alloys, besides having other interesting features, can lead to large magnetocrystalline anisotropies ${ }^{14}$ as well as to unconventional magnetic phase transitions as a function of temperature. ${ }^{42}$ In conjunction with these atomistic or nano-scale effects, it is important to understand the physics of magnetization reversal itself, in particular, because magnetization reversal measurements are frequently used to characterize and classify magnetic materials.

The magnetization reversal process by itself is already a rather complex process, which is determined by the non-trivial free energy landscape of a ferromagnet. As a consequence of this complexity, magnetization reversal may follow different routes ${ }^{54}$ such as multi domain (MD) formation, ${ }^{20,24,30,31,39,40}$ coherent uniform rotation, isolated domain nucleation followed by domain wall depinning and rapid propagation,,${ }^{17,49,51}$ or a sequential combination of these processes. However, a common characteristic of thin films and their magnetization reversal is that in order to minimize the magnetostatic energy, the magnetization tries to orient within the film plane, at least in the absence of large out-of-plane magnetocrystalline anisotropy $^{39}$ or other external driving forces. In the case of 
large out-of-plane magnetocrystalline anisotropy, it has been previously observed that it can be energetically favorable to form complex domains, such as stripe and bubble domains with a local magnetization component out-of-plane. ${ }^{24,30,39,40}$

Despite a large number of studies on magnetization reversal in thin films, many aspects of it are still not well understood because magnetization reversal is a collective phenomenon, just like ferromagnetism itself, but in addition it is also very strongly influenced by local property variations, which modify the already complex energy landscape and thus the specific reversal paths that magnetic systems can follow. ${ }^{62,63}$ Due to this complexity, the vast majority of studies has been focused on thin film systems, in which the correlation length of the reversal is very long and magnetization is reversed in a collective or uniform way. This can typically be achieved by means of good crystalline order. ${ }^{4,18,21,25,42,48,51}$ It is hereby important to remember that magnetic anisotropy depends strongly on the crystallographic order ${ }^{64}$ due to the quantum-mechanical spin-orbit interaction. Correspondingly, magnetization orientations along certain crystallographic axes are energetically favorable. ${ }^{65}$ Therefore, good crystalline order samples exhibit a well-defined and homogenous magnetocrystalline anisotropy, which leads to simpler energy landscapes and collective magnetization reversal modes. Films with good crystalline order are usually achieved by epitaxial growth on single crystal substrates, and indeed, many "textbook" cases of rather simple and predictable magnetization reversal behavior have been observed and classified in such samples. ${ }^{4,19,51}$ For instance, in this type of samples, the Stoner Wohlfarth (SW) model is widely used in order to extract magnetocrystalline anisotropy values. ${ }^{19}$

The opposite extreme of macroscopically ordered single crystal films, i.e., magnetically separated grains, in which every grain follows an individual and isolated path during magnetization reversal, has also been studied in detail. ${ }^{20,24,31,54,55,66}$ Here, the interest is primarily motivated by technological relevance because today's ultra high information storage densities in magnetic recording are fundamentally connected to the ability of achieving a strong localization of individual magnetization reversal processes. ${ }^{67-69}$ Also in this case, an accurate and predictable understanding of the magnetization reversal has been achieved, specifically by means of simple averaging over large numbers of individual grain magnetization reversals, for which interactions can be represented as mean-field effects. ${ }^{3,6-8,12,54}$ This means that the extreme cases of diverging and nano-scale limited magnetization reversal correlation are well understood and can be described with high accuracy.

The intermediate range of partial magnetization correlation is, however, far more difficult to understand. So, even though many works have analyzed how different types of local property variations affect magnetization reversal, ${ }^{1,13,15,27,28,33-38,44,45,52,57,66}$ the complexity of the underlying physics makes it very difficult to develop a comprehensive understanding of all the aspects involved. One such example is the effect of anisotropy dispersion that has been investigated in numerous studies. ${ }^{1,13,15,28,33-35,45,57}$ For instance, the angular dispersion impact onto perpendicular media was studied in Refs. 34 and 35 by extracting anisotropy dispersion angles from remanent magnetization data and correlating these values with X-ray diffraction (XRD) measurements. However, while in Ref. 34 the authors found that the anisotropy dispersion angle is narrower than the X-ray diffraction rocking curve width, the study in Ref. 35 found exactly the opposite. Another example is given by the attempt to correlate anisotropy dispersion with magnetization reversal for in-plane magnetized films. ${ }^{1,13,15,28,33,45,57}$ Also in this case, a clear correlation in between sample structure and magnetization reversal behavior could not be established, even though some of these works managed to correlate different magnetic properties with each other. Thus, a comprehensive and systematic analysis of the influence of crystallographic ordering onto magnetization reversal, especially in the regime of a sizable, but not diverging reversal correlation length is still lacking despite many decades of research on magnetization reversal in thin magnetic films.

The present work is intended to make a substantial step forward by devising a sample fabrication scheme that allows for a robust, reproducible, and quantifiable variation of crystallographic dispersion, and by means of magnetic characterization measurements develop an understanding of magnetization reversal in partially disordered thin films. To achieve this goal, we have devised a fabrication procedure that allows us to tune the crystallography of our samples and thus the anisotropy dispersion degree in a well-defined manner and observe its influence onto ferromagnetic properties, and specifically the magnetization reversal process. With this methodology, we managed to control local variations of the crystallographic structure in a continuous fashion from highly ordered samples, grown fully epitaxial to strongly disordered samples with limited or no texture.

The paper is organized as follows. In Sec. II, we motivate and introduce the specific material system chosen for this study, as well as the procedure to modify the crystallographic order and lateral uniformity. The structural and topographical characterizations have been performed by Xray diffractometry and atomic force microscopy (AFM). Section III describes the macroscopic magnetization reversal analysis of our samples, which we carried out by means of magneto optical Kerr effect (MOKE) measurements. Hereby, we also find an anomalous magnetization reversal behavior that occurs for partially disordered, but still anisotropic samples in the vicinity of the hard axis (HA). Specifically, this anomalous magnetization reversal behavior is characterized by the occurrence of substantial hysteresis as well as the non-monotonic behavior of $M_{r}$ and $H_{c}$ with respect to the applied field angle as one approaches the nominal hard axis. While this HA anomaly has been observed in the previous studies, it had not been studied in detail and no clear correlation with the crystallographic disorder level was established. In Sec. IV, we study the origin of this anomaly by MOKE magnetometry, MOKE microscopy, and Brillouin light scattering (BLS) spectroscopy. While MOKE magnetometry and MOKE microscopy give complementary information about average magnetization and microscopic magnetization distributions, the BLS measures both simultaneously by analyzing the spin wave spectra width and position, which in turn allows for a consistency check of all 
experimental observations. Also, we explain and mimic the occurrence of the anomaly with the help of a coupled two grain SW model ${ }^{70}$ and find excellent qualitative and even quantitative agreement in between the model simulations and our experimental data. Section V shows our microscopic study of the magnetization reversal process as a function of the applied field angle, which we performed by using MOKE microscopy. Hereby, our analysis has been focused on studying how grain orientation disorder below the domain wall length scale is related to non-uniform magnetization states. Specifically, we have studied the stability range of nonuniform magnetization states during magnetization reversal for films that exhibit different levels of crystallographic disorder and thus anisotropy dispersion. We observe a clear correlation in between the occurrence of domain states and the crystallographic order as well as with the angular dependence of the coercive field $H_{c}$, i.e., one of the key macroscopic measures of magnetization reversal.

\section{SAMPLE GROWTH AND STRUCTURAL CHARACTERIZATION}

In this work, we study primarily the regime of low crystallographic disorder and thus small dispersions of the anisotropy. This has the advantage that one has a well-defined reference point, namely, epitaxial single crystal films, which have long-range crystalline coherence and correspondingly exhibit long-range correlation of their magnetization reversal behavior.

Furthermore, our material system selection was based on the idea that we would utilize a film type, in which the magnetization reversal is very strongly and directly connected to the crystalline structure, so that variations in the crystallographic order would cause clearly visible and identifiable modifications of the magnetization reversal behavior. For this reason, we chose a uniaxial material system with an in-plane easy axis (EA). Uniaxial ferromagnetic systems show a particularly strong change in magnetic properties and magnetization reversal as one moves away from the crystallographically defined easy axis, and the in-plane orientation suppresses the relevance of magnetostatic interactions, which can blur or even mask the direct impact of the crystalline orientation onto magnetization reversal. ${ }^{48}$

In order to achieve the desired uniaxial in-plane magnetic anisotropy, we chose Co as the film material and selected the (1010) surface orientation, which has the magnetically easy axis parallel to the [0001] crystallographic axis, lying in the film plane. For the film growth of this reference film system, we followed the work by Yang et al. ${ }^{71}$ and adapted their recipe to achieve optimum sample growth conditions in our ultra high vacuum (base pressure of $10^{-8}$ Torr) sputter deposition system. The overall growth sequence and the epitaxial relations of our base material system are shown in Fig. 1. As substrates, we used Si wafers with (110) orientation. The native oxide on the wafers was removed by using a wet hydrofluoric acid (HF) chemical etch, which we monitored by means of ellipsometric measurements. Specifically, a solution of $2.5 \%$ $\mathrm{HF}$ in deionized water was used at room temperature for 5 min, after which the substrate was immediately transferred (a)

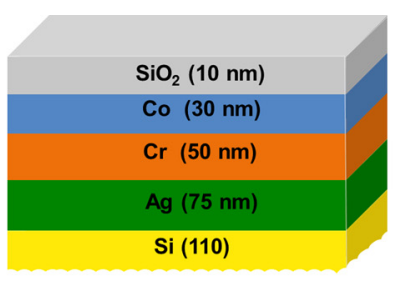

(b)

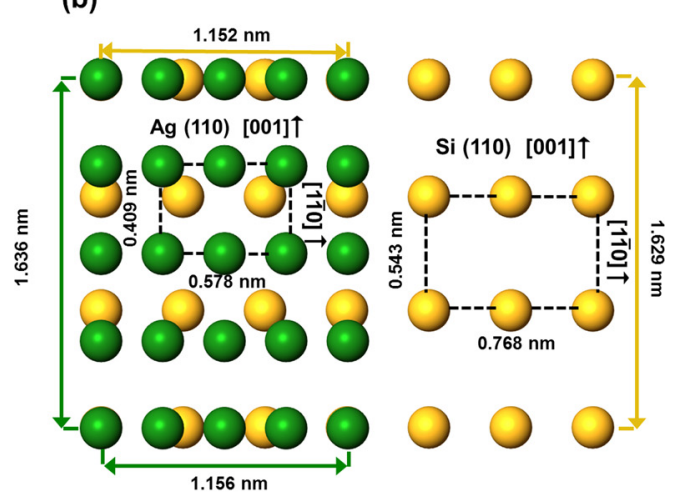

(c)

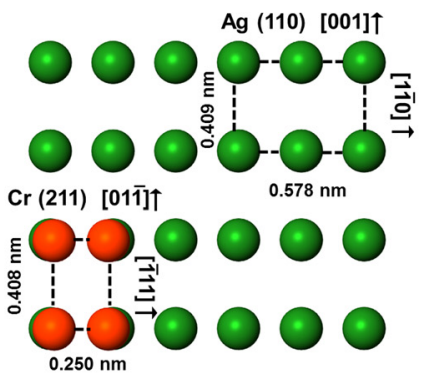

(d)

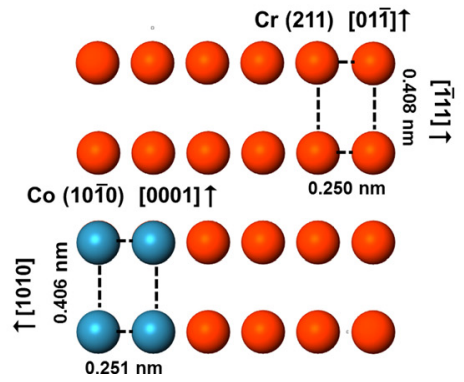

FIG. 1. (a) Schematic of the layer sequence used to obtain epitaxial growth of Co (1010) with in-plane uniaxial magnetic anisotropy; (b)-(d) display the epitaxial - relations between subsequent layers in the stacking sequence $\mathrm{Si}(110)[001] / \operatorname{Ag}(110)[001] / \operatorname{Cr}(211)[0 \overline{1} 1] / \operatorname{Co}(10 \overline{1} 0)[0001]$ by means of their respective crystallographic surface unit cells.

into the UHV sputter system. On top of the so-prepared $\mathrm{Si}$ (110) single crystal wafers, $75 \mathrm{~nm}$ of $\mathrm{Ag}$ and $50 \mathrm{~nm}$ of $\mathrm{Cr}$ layers were deposited to facilitate the desired epitaxial growth of the Co layer. Subsequently, Co films of $30 \mathrm{~nm}$ thickness were deposited by DC magnetron sputtering onto this template layer sequence. On top of the Co-film, $10 \mathrm{~nm}$ of $\mathrm{SiO}_{2}$ was RF sputtered to prevent the Co from oxidizing under ambient conditions. All layers were grown at room temperature using an Ar-gas sputter pressure of 3 mTorr.

The crystallographic relationship between layers based on the respective bulk lattices can be seen in Figs. 1(b) $-1(\mathrm{~d}){ }^{71} \mathrm{Si}$ has a face-centered diamond crystal structure with a unit cell length of $0.543 \mathrm{~nm}$, so that the Si (110) 
crystallographic plane results in a rectangular cell with dimensions of $0.768 \mathrm{~nm} \times 0.543 \mathrm{~nm}$ as indicated in Fig. 1(b). $\mathrm{Ag}$ has a face centered cubic (fcc) crystallographic structure with a unit cell length of $0.409 \mathrm{~nm}$, resulting in the $\mathrm{Ag}$ (110) crystallographic orientation having a rectangular unit cell with $0.578 \mathrm{~nm} \times 0.409 \mathrm{~nm}$ dimensions [Fig. 1(b)]. Therefore, a $2 \times 4 \mathrm{Ag}$ (110) supercell mesh has the dimensions of $1.156 \mathrm{~nm} \times 1.636 \mathrm{~nm}$, which matches almost perfectly the $1.152 \mathrm{~nm} \times 1.629 \mathrm{~nm}$ size of half a $3 \times 3 \mathrm{Si}(110)$ supercell mesh, resulting in a lattice mismatches of only $0.4 \%$ along the $\mathrm{Si}$ (110) [001]/Ag (110) [001] direction and 0.35\% along the $\mathrm{Si}$ (110) [110]/Ag (110) [110] direction [Fig. 1(b)]. The subsequently grown $\mathrm{Cr}$ with its body centered cubic (bcc) crystallographic structure fits well in (211) surface orientation onto $\mathrm{Ag}$ (110), as can be seen in Fig. 1(c), having a lattice mismatch of only $-0.25 \%$ in the $\mathrm{Ag}(110)[001] / \mathrm{Cr}(211)[0 \overline{1} 1]$ direction, even though this creates a not insubstantial mismatch in the $\mathrm{Ag}(110)[110] / \mathrm{Cr}$ (211)[1̄11] direction of $-13.5 \%$. However, as corroborated by X-ray diffractometer measurements [see Fig. 2(a)], this
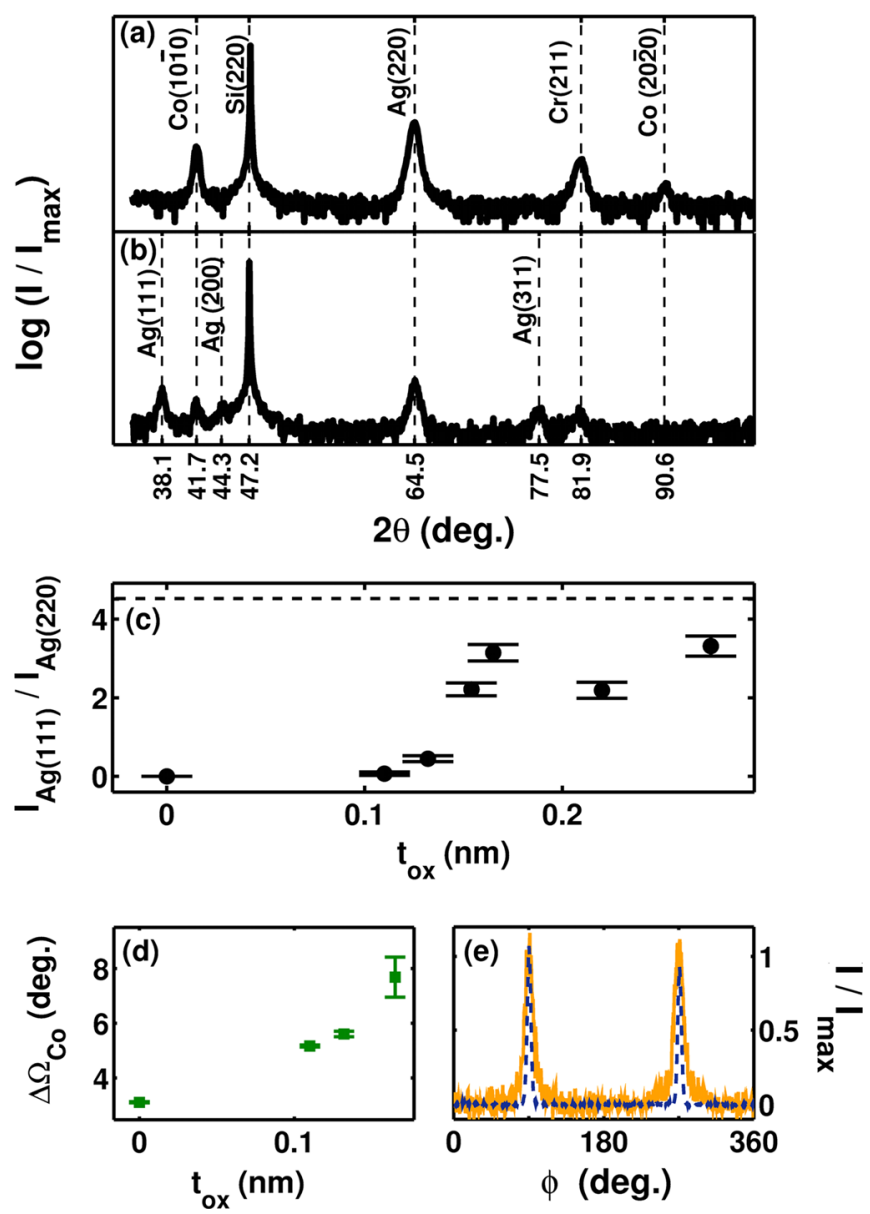

FIG. 2. Structural data for the sample series studied here: $\theta-2 \theta \mathrm{X}$-ray diffraction spectra are shown for the fully epitaxial sample $t_{o x}=0 \mathrm{~nm}$ (a) and for a partially epitaxial sample with $t_{o x}=0.132 \mathrm{~nm}$ (b). (c) displays the ratio between the intensities of the $\operatorname{Ag}(111)$ and $\operatorname{Ag}(220)$ diffracted peaks as a function of $\mathrm{SiO}_{2}$ interlayer deposition thickness $t_{o x}$. The dashed line in (c) represents the expected $\mathrm{Ag}(111) / \mathrm{Ag}(220)$ peak-ratio for randomly oriented polycrystalline Ag. (d) shows the (1010)Co rocking curve width $\Delta \Omega_{C o}$, given by its full width at half maximum, as a function of $t_{o x}$. X-ray diffraction $\Phi$ scan measurements of the Co (1011) pole for samples with $t_{o x}=0 \mathrm{~nm}$ (dashed line) and $t_{o x}=0.132 \mathrm{~nm}$ (solid line) are presented in (e). considerable mismatch does not prohibit the epitaxial growth of $\mathrm{Cr}$ with (211) orientation onto the $\mathrm{Ag}$ (110) template layer. Finally, the $\mathrm{Cr}$ (211) surface provides an excellent template for Co with hexagonal close packed (hcp) crystallographic structure and (1010) surface orientation, aligning the [0001] direction of Co along the [011] direction of the $\mathrm{Cr}$ (211) surface with a mismatch of only $-0.5 \%$ in this specific

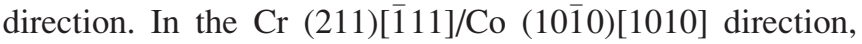
the mismatch is only $0.4 \%$ [Fig. 1(d)]. In this specific surface orientation, the crystallographic c-axis of Co is in the film plane and given that our films have near bulk-like crystallography, the c-axis shows indeed typical easy axis behavior.

In order to characterize the crystallographic structure of our films, we performed XRD measurements in $\theta-2 \theta$ geometry as well as rocking curve measurements and $\Phi$ scans. Figure 2(a) shows a typical $\theta-2 \theta$ XRD measurement for a fully epitaxial sample. The data are normalized to the highest intensity, given by the Si (220) substrate peak value. Here, one only observes $\mathrm{Si}$ (220), Ag (220), Cr (211), Co (1010), and Co $(20 \overline{2} 0)$ diffraction peaks, corroborating the epitaxial nature of our samples as well as the previously discussed epitaxial growth sequence. The $\mathrm{SiO}_{2}$ overlayer grows amorphously and, thus, it does not show any diffracted peak in XRD measurements.

Using the epitaxial growth sequence as a starting point for our sample fabrication and overall study, we have grown partially epitaxial samples with varying degree of crystallographic disorder. In order to achieve a continuous and well defined modification of the crystallographic structure from full epitaxy with (1010) Co orientation to samples with very limited grain orientation alignment and uniformity, we have partially interrupted the epitaxy by depositing a nonepitaxial $\mathrm{SiO}_{2}$ layer directly onto the $\mathrm{HF}$ etched $\mathrm{Si}$ substrate. The $\mathrm{SiO}_{2}$ interlayer has been deposited by $\mathrm{RF}$ magnetron sputtering in our UHV sputter deposition system at low plasma power in order to achieve precise thickness control. For this purpose, we have chosen a deposition power of $60 \mathrm{~W}$ and an Ar pressure of 3 mTorr. Under these conditions, the deposition rate of $\mathrm{SiO}_{2}$ is very low, $0.011 \mathrm{~nm} / \mathrm{s}$, so that it opens up a substantial deposition time window to conduct a precise and reproducible study of partial epitaxy suppression. For our study, we grew a set of 15 samples with different $\mathrm{SiO}_{2}$ interlayer thicknesses $\left(t_{o x}\right)$. In this work, however, we mainly focus on samples with $t_{o x}=0 \mathrm{~nm}, 0.11 \mathrm{~nm}, 0.132 \mathrm{~nm}$, $0.154 \mathrm{~nm}, 0.165 \mathrm{~nm}$, and $0.275 \mathrm{~nm}$, because they present the $t_{o x}$-range, in which the magnetic properties are affected in the most relevant way.

XRD measurements in $\theta-2 \theta$ geometry for one of our partially epitaxial samples with $t_{o x}=0.132 \mathrm{~nm}$ can be seen in Fig. 2(b). Also here, the data are normalized to the $\mathrm{Si}$ (220) peak intensity. In addition to the peaks that correspond to the epitaxial growth sequence, one can also observe peaks that correspond to Ag (111), (200), and (311) crystallographic orientations, showing that this Ag-film is neither single crystalline nor epitaxial. However, this particular sample is not crystallographically random either. For polycrystalline $\mathrm{Ag}$ with random grain orientations, the (111) peak intensity has 4.50 times the intensity of the $\mathrm{Ag}$ (220) peak. ${ }^{72}$ However, the $t_{o x}=0.132 \mathrm{~nm}$ sample shows a $\mathrm{Ag}$ (220) peak 
intensity that is higher than that for the $\mathrm{Ag}$ (111) peak, which means that this sample has a very substantial degree of (220) texture. Given that a predominant Ag (220) texture does not grow on a completely amorphous template layer, the texture observed in Fig. 2(b) must be the result of a still existing partial epitaxy, which cannot be fully suppressed by the only $0.132 \mathrm{~nm}$ thick Si-oxide. The partial epitaxy nature of our samples and the increased suppression of epitaxy by increasing $t_{o x}$ can be seen clearly in Fig. 2(c). Upon increasing the Si-oxide interlayer thickness, the ratio between the Ag (111) and Ag (220) peak intensities increases, i.e., we observe a gradual decrease of the $\mathrm{Ag}$ (220) texture [Fig. 2(c)]. Therefore, as we introduce the $\mathrm{SiO}_{2}$ interlayer, we interrupt the epitaxy and we gradually change the template from a pure Ag (110) single crystal layer towards a randomly orientated polycrystalline Ag layer. As one modifies the Ag crystallographic structure, the crystallographic orientation of the subsequent Co-film is also tuned in a similar fashion. The increased suppression of crystalline alignment in the Co-film upon increasing $t_{o x}$ has also been monitored by measuring the rocking curves of the Co (1010) peak. Consistent with the XRD results in $\theta-2 \theta$ geometry, we find a continuous increase of the Co (1010) peak width upon increasing the Si-oxide interlayer thickness, measured as the full width half maximum $\left(\Delta \Omega_{C o}\right)$ [Fig. 2(d)]. While for the epitaxial sample with $t_{o x}=0 \mathrm{~nm}, \Delta \Omega_{C o}$ is $3.13^{\circ} \pm 0.02^{\circ}$, the sample with $t_{o x}=1.32 \mathrm{~nm}$, for instance, exhibits a $\Delta \Omega_{C o}$ of $5.61^{\circ} \pm 0.10^{\circ}$. As $t_{o x}$ increases further, $\Delta \Omega_{C o}$ increases as well, indicating a continued decrease of crystallographic alignment. Furthermore, in order to measure the azimuthal crystallographic spread of the grains in the Co layer as a function of $t_{o x}$, $\Phi$ scans have been measured on the Co (10)11) pole. Figure 2(e) displays two examples of these measurements, namely, for the sample with $t_{o x}=0 \mathrm{~nm}$ (dashed line) and for the sample with $t_{o x}=0.132 \mathrm{~nm}$ (solid line). For both samples, two clear diffraction peaks can be observed that are $180^{\circ}$ apart from each other, thus demonstrating that the crystals are highly oriented in our samples. In addition, we can observe that the width of the diffracted peaks increases with increasing $t_{o x}$, which verifies the growing azimuthal spread of the Co grain orientations for larger $t_{o x}$, a fact that is consistent with the other structural measurements.

To test, if the change in crystallographic alignment due to partial epitaxy suppression affects the growth in other ways as well, we also measured the root mean square (RMS) surface roughness for all samples by AFM. We observed no change in the film roughness upon introducing the Si-oxide interlayer. Instead, all samples show RMS roughness at a nearly identical level of $1.1 \mathrm{~nm}$. This finding means that the structural changes in our sample series are clearly dominated by a change in crystallographic grain alignment, introduced by a partial interruption of epitaxy by means of a $\mathrm{SiO}_{2}$ interlayer.

\section{BASIC MAGNETIC CHARACTERIZATION}

The macroscopic magnetic properties have been characterized by means of a home built MOKE setup. Figure 3 shows a schematic of our experimental MOKE setup. The

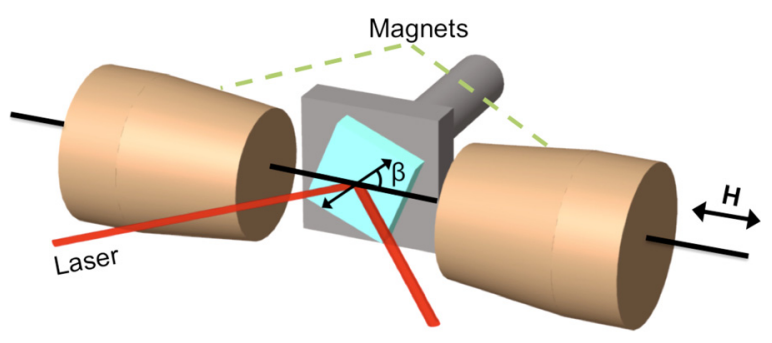

FIG. 3. Schematic of our experimental setup, which allows for an azimuthal sample rotation with respect to the applied magnetic field axis within our MOKE magnetometer. $\beta$ defines the angle between the easy axis of magnetization and applied field direction.

sample is mounted onto a holder that can be continuously rotated around the surface normal with a precision of better than $0.5^{\circ}$, which allows for precise measurements of the inplane angular dependence of magnetic properties. Here, we define $\beta$ as the angle between the applied field and the EA of our sample. The magnetic poles of an electromagnet were placed near the sample and were fixed throughout the experiment. The plane of incidence was aligned with the applied field direction and consequently with the magnetization vector in saturation, an arrangement which is conventionally referred to as longitudinal MOKE geometry.

Figure 4 shows several hysteresis loops for the fully epitaxial sample [Figs. 4(a)-4(c)] and a partially epitaxial sample with $t_{o x}=0.132 \mathrm{~nm}$ [Figs. 4(d)-4(f)] for different applied field angles with respect to the samples' easy axes. Specifically, figures 4(a) and 4(d) display hysteresis loops for the applied field being oriented along the EA $\left(\beta=0^{\circ}\right)$, figures 4(b) and 4(e) for $\beta=40^{\circ}$, and figures 4(c) and 4(f) at $\beta=60^{\circ}$. In each case, the sample magnetization $(M)$ is

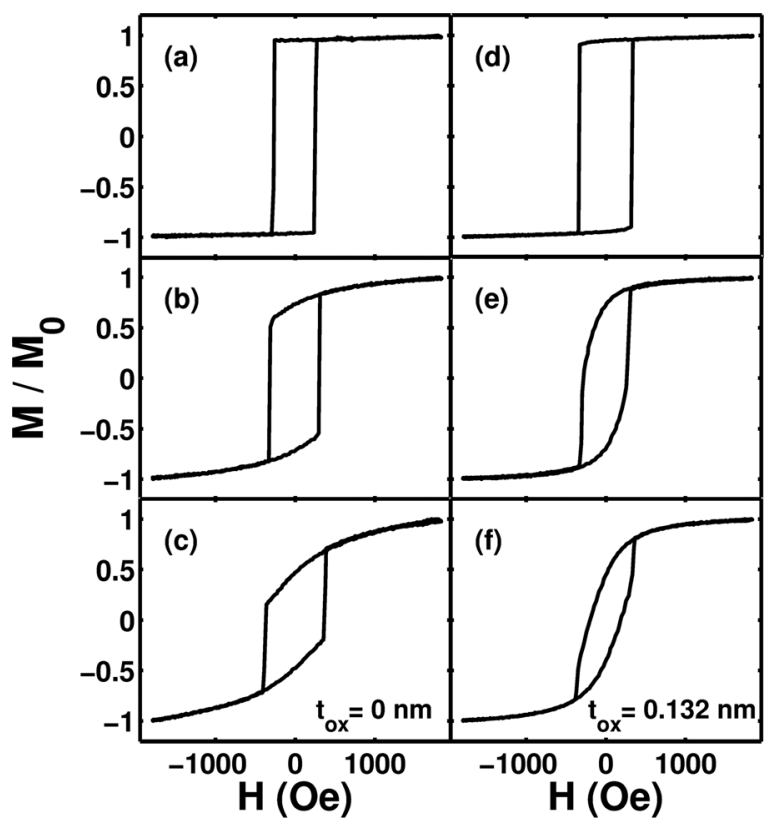

FIG. 4. Hysteresis loop measurements for the fully epitaxial sample with $t_{o x}=0 \mathrm{~nm}$ (a)-(c) and for the partially epitaxial sample with $t_{o x}=0.132 \mathrm{~nm}$ (d)-(f). (a) and (d) show hysteresis loops with the applied field oriented along the nominal EA of magnetization. (b) and (e) show hysteresis loops for an applied field orientation of $40^{\circ}$ away from the EA, and (c) and (f) at $60^{\circ}$ away from the nominal EA. 
normalized to a reference magnetization $M_{0}$, which is the magnetization value at the maximum applied field of $H=1800$ Oe. $^{79,80}$

Along the EA, both samples $\left(t_{o x}=0 \mathrm{~nm}\right.$ and $t_{o x}$ $=0.132 \mathrm{~nm}$ ) show a very similar behavior with high remanent magnetization and a sample size avalanche magnetization reversal [Figs. 4(a) and 4(d)]. Despite these similarities, one can also observe differences. For instance, the sample with $t_{o x}=0.132 \mathrm{~nm}$ exhibits a weak bending of the hysteresis loop just before the avalanche reversal point is reached, an effect that is absent or at least far weaker in the epitaxial sample. This behavior is caused by the fact that with only partial epitaxy, the entire film is no longer a single crystal and the anisotropy axes of different grains are not fully aligned anymore, i.e., there is visible anisotropy dispersion. Thus, even for field orientations along the nominal EA, magnetization rotation is occurring and becomes visible just prior to the correlated magnetization reversal switch. As the external field is applied away from the EA, i.e., for $\beta=40^{\circ}$ [Figs. 4(b) and 4(e)] and $\beta=60^{\circ}$ [Figs. 4(c) and 4(f)], the hysteresis loops change gradually for both samples. But even though both samples exhibit a clear angular dependence and a clear transition from EA to HA behavior, the variation of hysteresis loops as a function of $\beta$ differs between the two samples. For instance, at $\beta=60^{\circ}$, the reversal of the fully epitaxial sample is still dominated by an abrupt magnetization switch [Fig. 4(c)], while for the partially epitaxial sample, the hysteresis loop shows a far bigger bending and a much smaller magnetization switch, giving the hysteresis loop an overall far more gradual transition appearance [Fig. 4(f)]. ${ }^{53}$

In order to achieve a more detailed characterization of the angular dependence of magnetization reversal properties in our sample series, we have measured hysteresis loops as a function of $\beta$ in steps of $5^{\circ}$ or even $0.5^{\circ}$ in the vicinity of the HA. From these hysteresis loop measurements, we have extracted core reversal quantities, such as, for instance, the remanent magnetization $M_{r}$.

Figures 5(a) $-5(\mathrm{~g})$ show $M_{r}$ values as a function of $\beta$ for 7 different samples, representing different $t_{o x}$ and thus different degrees of anisotropy dispersion. The remanent magnetization value for each $\beta$ is calculated by averaging the absolute values of the positive and negative remanent magnetization over 20 loops measured under identical conditions. Similar to the hysteresis loops shown in Fig. 4, one observes a strong angular dependence of $M_{r} / M_{0}$ for all samples with a periodicity of $180^{\circ}$, which is the signature of uniaxial anisotropy, except for the sample with the thickest oxide interlayer, $t_{o x}=0.55 \mathrm{~nm}$, for which $M_{r}$ does not show any orientation dependence. One can also appreciate from the series of curves that the angular dependence becomes slightly less pronounced as $t_{o x}$ increases, even before it collapses entirely at $t_{o x}=0.55 \mathrm{~nm}$.

In the case of the fully epitaxial sample and for $t_{o x}=0.11 \mathrm{~nm}$, the remanent magnetization is almost exactly 1 along the $\mathrm{EA}\left(\beta=0^{\circ}\right)$ and its value decreases coherently as we go away from the EA towards the HA $\left(\beta=90^{\circ}\right)$, where $M_{r}$ vanishes as expected. This behavior agrees very well with the ideal case of the $\mathrm{SW}$ model, ${ }^{70}$ which is shown as a dotted-dashed line in Figs. 5(a)-5(g).
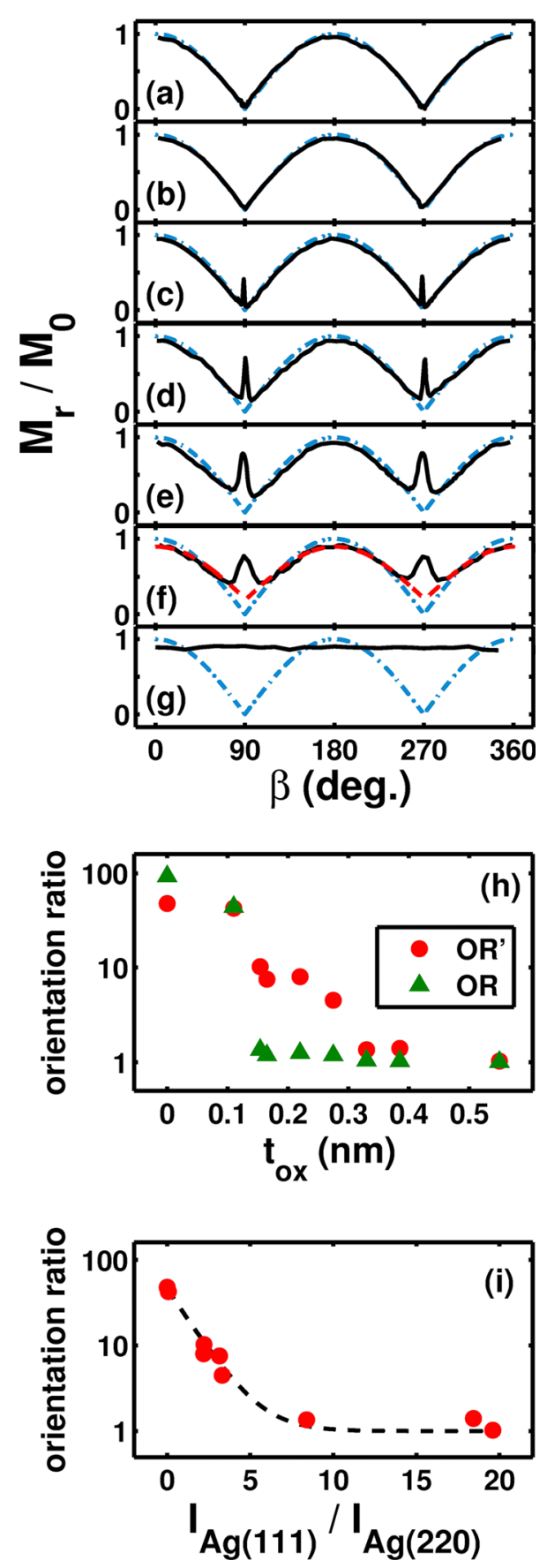

FIG. 5. Magnetic properties of the partially epitaxial Co-film sample series. The applied field orientation dependence of the remanent magnetization, normalized to $M_{0}$, is displayed in (a)-(g) for samples with different $t_{o x}$ : (a) $0 \mathrm{~nm}$, (b) $0.11 \mathrm{~nm}$, (c) $0.132 \mathrm{~nm}$, (d) $0.154 \mathrm{~nm}$, (e) $0.165 \mathrm{~nm}$, (f) $0.275 \mathrm{~nm}$, and $(\mathrm{g}) 0.55 \mathrm{~nm}$. The solid line represents the measured $M_{r} / M_{0}$ data, while dotted-dashed line is the geometric projection of the EA magnetization onto the applied field axis. The dashed line in (f) is the least-squares fit of Eq. (2) to the experimental data. (h) shows the extrapolated orientation ratio $\left(O R^{\prime}\right)$ (dots) and conventional or two point orientation ratio (OR) (triangles) as a function of $t_{o x}$. (i) displays $O R^{\prime}$ as a function of the $\mathrm{Ag}(111) / \mathrm{Ag}(220)$-peak ratio from the $\mathrm{X}$-ray diffraction data, with the dashed line being a guide to the eye.

For samples with higher disorder, i.e., $t_{o x}=0.132 \mathrm{~nm}$, $0.154 \mathrm{~nm}, 0.165 \mathrm{~nm}$, and $0.275 \mathrm{~nm}$ [Figs. 5(c)-5(f)], one still observes a strong variation of $M_{r} / M_{0}$ as a function of $\beta$. Also, for most of the $\beta$ range, the angular dependence of $M_{r}$ seems only moderately or even minimally affected, as if the 
decrease in crystalline alignment had little influence onto the remanent magnetization states. However, for applied field orientations near the HA, the behavior is drastically changed in a non-trivial fashion. Instead of a vanishing or only small remanence value, as expected for simple uniaxial samples, one observes a steep increase of the $M_{r} / M_{O}$ values along the nominal HA upon decreasing the crystalline alignment. The second aspect that is very unusual is the fact that this high HA remanence is limited to the immediate vicinity of the HA itself, resulting in very sharp $M_{r}$-peaks in Figs. 5(c)-5(f). This unusual behavior is discussed in more detail in Sec. IV.

One approach to quantify partial or imperfect uniaxial magnetic anisotropy is the measurement of the orientation ratio of a sample. ${ }^{73,74}$ Conventionally, the orientation ratio $O R$ is defined as

$$
O R=\frac{M_{r e a}}{M_{r h a}}
$$

where $M_{\text {rea }}$ and $M_{\text {rha }}$ are the remanent magnetization values measured along the EA and the HA, respectively. In our subsequent analysis, however, we have modified the conventional orientation ratio analysis to make it more robust, because the conventional formulation Eq. (1), is based on a two-point measurement only $\left(M_{\text {rea }}\right.$ and $\left.M_{r h a}\right)$. Instead, we consider $M_{r} / M_{O}$ data in the entire field orientation range $(\beta)$ by using an adapted SW formula

$$
\frac{M_{r}}{M_{o}}(\beta)=c+a|\cos (\beta)| .
$$

Hereby, $c$ and $a$ are parameters that define the degree of uniaxial alignment. This equation is capable of describing the full spectrum from perfect uniaxial alignment to isotropic samples by changing the $c$ and $a$ parameters. We have performed least-squares fits to Eq. (2) for all experimental $M_{r} / M_{O}$ data sets. In Fig. 5(f), we show, as an example, the fit for the $t_{o x}=0.275$ sample by a dashed line. The least square fits agree very well with all experimental data, except in the vicinity of the nominal HA for the samples with $t_{o x}=0.132$ $\mathrm{nm}, 0.154 \mathrm{~nm}, 0.165 \mathrm{~nm}$, and $0.275 \mathrm{~nm}$. Here, the HA anomaly appears, which cannot be described with the simple sinusoidal functional form, Eq. (2).

From the fit parameters $a$ and $c$ in Eq. (2), we have calculated the extrapolated magnetic orientation ratio $\left(O R^{\prime}\right)$, which is given as the ratio of the extrapolated remanent magnetizations in the EA $\left(M^{\prime}{ }_{r e a}\right)$ and HA $\left(M^{\prime}{ }_{r h a}\right)$

$$
O R^{\prime}=\frac{M_{r e a}^{\prime}}{M_{r h a}^{\prime}}=\frac{a+c}{c}
$$

The conventional orientation ratio $(O R)$ and extrapolated orientation ratio $\left(O R^{\prime}\right)$ values are displayed in Fig. 5(h) as a function of the $\mathrm{SiO}_{2}$ interlayer thickness by green triangles and red circles, respectively. For the fully epitaxial sample as well as for the $t_{o x}=0.11 \mathrm{~nm}$ sample, $O R$ and $O R^{\prime}$ values are extremely high, consistent with the high level of crystallographic alignment in these samples. As the crystallographic disorder increases, both $O R$ and $O R^{\prime}$ values decrease. However, they do so differently. Only for samples with $t_{o x}=0.33 \mathrm{~nm}$ or higher, $O R$ and $O R^{\prime}$ are nearly equal and close to 1 , indicating isotropic or nearly isotropic behavior. For intermediate crystallographic disorder levels, one can observe the weakness of the conventional orientation ratio $(O R)$ definition. In samples where the HA anomaly occurs, the $O R$ value drops suddenly to almost 1 , even though the samples are clearly anisotropic in their overall $M_{r} / M_{O}$ vs. $\beta$ dependence. This occurs because $O R$ is directly impacted by any anomaly occurring along the HA or EA, even if it afflicts only a very small $\beta$-range. On the other hand, the continuous decrease of uniaxial magnetic anisotropy upon increasing $t_{o x}$ can be very well monitored by means of the extrapolated orientation ratio $\left(O R^{\prime}\right)$ because in contrast to the conventional $O R$, the extrapolated orientation ratio $\left(O R^{\prime}\right)$ is based on the entire field orientation dependence of the remanent magnetization and not only on two specific values.

Figure 5(i) shows the measured $O R^{\prime}$ values as a function of the XRD peak ratio $\left(I_{\mathrm{Ag}(111)} / I_{\mathrm{Ag}(220)}\right)$. This plot shows very clearly that the magnetic anisotropy alignment in the Co-films is fully correlated with the degree of (220) texture in the Ag-template layers. This is, of course not a surprising result, because it is the (220) Ag texture that enables the growth and the alignment of the Co (1010) surface orientation. Thus, Figure 5 demonstrates very clearly that our methodology of partially interrupting epitaxy allows for the controlled and reproducible introduction of crystalline misalignment that simultaneously affects the magnetic anisotropy dispersion.

\section{ANOMALOUS MAGNETIZATION REVERSAL NEAR THE HA}

Signatures of anomalies in the magnetization reversal process, i.e., the non-monotonic behavior of $M_{r}$ and $H_{c}$ as a function of the applied field angle, have been previously observed near the magnetic hard axis by several groups in samples with anisotropy dispersion, ${ }^{13,15,38,57}$ including samples with biaxial anisotropy. ${ }^{36,44,52}$ However, in these previous works, the phenomenon was not explored in detail and the underlying physics of this anomaly could not be clearly identified. In Ref. 13, the authors made an experimental observation of the anomaly by detecting hard axis hysteretic behavior while applying biased circular field cycles. This paper also describes a method of measuring the applied field angle range, in which the HA anomaly appears, which is assumed to be equal to the anisotropy dispersion angle. However, the described methodology does not allow any other quantitative observation, such as, the shape and overall the height of the anomalous magnetization, and furthermore there is no correlation with the crystallographic structure of the samples reported. In the works by Scheurer et al., ${ }^{15}$ Hamrle et al., ${ }^{36}$ Gaier et al., ${ }^{44}$ and Trudel et al., ${ }^{52}$ an increase of the coercive field was reported when the field was applied along the HA in biaxial magnetocrystalline anisotropy systems, specifically bcc Fe thin films ${ }^{15}$ and Co-based Heusler alloy thin films. ${ }^{36,44,52}$ In bcc Fe thin films, ${ }^{15}$ the increase of the $H_{c}$ field was associated to the occurrence of structural twin domains with slight lateral misalignment, but it was not 
investigated how this misalignment affects the anomalous phenomena in any quantitative or even qualitative manner. In the Co-based Heusler alloy works, the anomaly was associated to the pure cubic anisotropy ${ }^{36,44,52}$ and a checkerboard magnetic domain structure appearance during the magnetization reversal. However, none of these studies ${ }^{36,44,52}$ succeeded in correlating this anomaly with the crystal structure. In Refs. 45 and 57, the authors found an increase of the coercive field near the HA in a soft FeCo ferromagnetic thin film alloy. In these works, the authors associated the HA anomalous behavior with the anisotropy dispersion and they analyze the angular width and coercive field change in samples with different thickness and underlayer structure. Nevertheless, no detailed explanation of the phenomenon in terms of a microscopic picture was given and no correlation of the anomaly with the crystallographic quality was reported.

So, even though anomalous HA behavior was previously observed for a number of materials, none of these works was able to clearly correlate this effect with the actual crystalline structure, primarily due to the lack of a systematic tuning of crystallographic alignment. Thus, we have studied here the origin of the HA anomalous magnetization reversal in detail. We have reported on the existence of the HA anomaly previously, ${ }^{75}$ classified its occurrence in correlation with the crystallographic order in our samples, as well as given a qualitative argument for it by means of a simple model. In this work, we present our full analysis of this phenomenon and make quantitative comparisons with model calculations.

Figure 6 shows three hysteresis loops measured in the partially epitaxial sample with $t_{o x}=0.132 \mathrm{~nm}$ along the

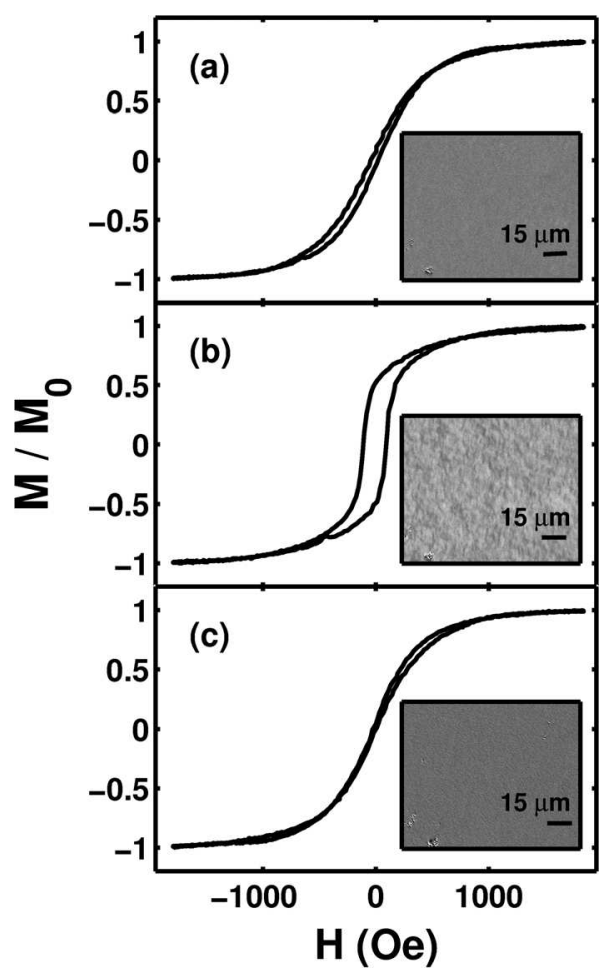

FIG. 6. Hysteresis loop data for a partially epitaxial sample with $t_{o x}=0.132 \mathrm{~nm}$, measured for field orientations of $\pm 2^{\circ}$ away from the HA, (a) and (c), respectively, and along the HA, displayed in (b). The insets show the respective remanent magnetization states acquired by means of Kerr microscopy.
HA [Fig. 6(b)] and $\pm 2^{\circ}$ away from the HA, i.e., at $\beta=88^{\circ}$ [Fig. 6(a)] and at $\beta=92^{\circ}$ [Fig. 6(c)]. From the hysteresis loop at $\beta=88^{\circ}$ [Fig. 6(a)], we see that the hysteresis effect has almost completely disappeared as expected for a perfect uniaxial sample. However, once the applied field is oriented exactly along the nominal HA, a substantial hysteresis effect occurs with considerable values for $M_{r}$ and $H_{c}$ [Fig. 6(b)]. If the field is applied along $\beta=92^{\circ}$, the nearly hysteresis free conventional hard axis behavior re-emerges by having almost vanishing values for $M_{r}$ and $H_{c}$ [Fig. 6(c)]. The insets in Figures 6(a)-6(c) show Kerr microscopy images of the remanent magnetization states taken after saturating the sample in the same applied field directions, i.e., $\beta=88^{\circ}, \beta=90^{\circ}$, and $\beta=92^{\circ}$. $^{81}$ The remanent magnetization pictures for applied fields $\pm 2^{\circ}$ away from the HA [insets in Figs. 6(a) and 6(c)] show uniform states. This is the expected behavior for a highly ordered uniaxial sample, because the magnetization reversal is dominated by a coherent rotation near the HA. However, an anomalous non-uniform magnetization state appears in the case of the magnetic field being applied exactly along the HA. This microscopic observation corroborates the hysteresis loop results and demonstrates that the magnetization reversal along the nominal HA is not dominated by a simple uniform magnetization rotation anymore as one would have expected for a simple uniaxial sample with perfect crystallographic alignment. We have performed the same remanent magnetization measurements by means of Kerr microscopy for the fully epitaxial sample, and as expected, in this case we only observe the conventional uniform magnetization state for all applied field directions. In our Kerr microscopy studies, we found the anomalous HA remanence, shown in Figs. 5(c)-5(f), to always correlate with the occurrence of a non-uniform remanent magnetic state. Moreover, from Fig. 5, one can also deduce that even though the crystallographic alignment is reduced by introducing a Si-oxide interlayer of thickness $t_{o x}=0.11 \mathrm{~nm}$, no HA anomaly is observed as indicated by the nearly identical curves in Figs. 5(a) and 5(b). Thus, the anomaly appears to be a threshold phenomenon that only occurs once a critical crystallographic misalignment and thus certain anisotropy dispersion has been surpassed. The progression of the anomaly behavior from Figs. 5(c) to 5(f) furthermore means that the anomalous remanent HA magnetization grows as a function of the crystalline misalignment angles, and it also occurs in a continuously increasing range of the field angle $\beta$.

In order to clarify the origin of this anomaly, we have devised a theoretical model for the purpose of describing the main features of our sample series. One of the principal properties of our samples is that they have uniaxial anisotropy, but are not a single crystal and that the crystallographic misalignment varies in a continuous fashion from sample to sample. Thus, the description of our samples requires a model that is capable of mimicking the crystalline misalignment and does so in a tunable way, while displaying an overall magnetic anisotropy. The simplest model that includes these characteristics is a two-grain SW model with misaligned anisotropy axes. ${ }^{82}$ Furthermore, it is clear that the 
grains we consider should be exchange coupled, because our films contain only $\mathrm{Co}$, so that even in between misaligned Co-grains, the exchange coupling must be strong.

A schematic of the model geometry can be found in Fig. 7. The magnetization unit vectors of the two grains are represented by $\hat{m}_{1}$ and $\hat{m}_{2}$, while the effective inter-granular exchange coupling constant is given as $J$. The two uniaxial grains are characterized by their respective first order anisotropy constants $K_{1}$ and $K_{2}$ as well as by the orientations of their magnetocrystalline easy axes, which are given by the unit vectors $\hat{n}_{1}$ and $\hat{n}_{2}$ that define a grain misalignment angle $\omega . \vec{H}$ represents the applied field. The nominal EA and HA are given by the averaged orientation of the grains' EA and HA vectors. Using this nomenclature, the total energy $E$ of the coupled two-grain system is ${ }^{83}$

$$
\begin{aligned}
E= & -J\left(\hat{m}_{1} \cdot \hat{m}_{2}\right)-\vec{H} \cdot\left(\hat{m}_{1}+\hat{m}_{2}\right)-\frac{1}{2} K_{1}\left(\hat{m}_{1} \cdot \hat{n}_{1}\right)^{2} \\
& -\frac{1}{2} K_{2}\left(\hat{m}_{2} \cdot \hat{n}_{2}\right)^{2} .
\end{aligned}
$$

In order to simplify the mathematical treatment and enhance the transparency of the model, we have made a number of additional assumptions and simplifications. First, identical magnetocrystalline anisotropies $(K)$ for both grains can be assumed, given that our films only contain one material, namely, Co. Furthermore, the local magnetization can be restricted to in-plane orientations under consideration of the in-plane magnetocrystalline anisotropy and the thin film nature of our samples, which create a strong demagnetizing effect along the surface normal. Under these simplifying assumptions, we find that the total energy is given by

$$
\begin{aligned}
E= & -J \cos \left(\beta_{1}-\beta_{2}\right)-H\left[\cos \left(\beta_{1}-\beta\right)+\cos \left(\beta_{2}-\beta\right)\right] \\
& -\frac{1}{2} K\left[\sin ^{2}\left(\beta_{1}-\frac{\omega}{2}\right)+\sin ^{2}\left(\beta_{2}+\frac{\omega}{2}\right)\right],
\end{aligned}
$$

where $\beta_{1}$ and $\beta_{2}$ are the in-plane orientation angles of the corresponding magnetization vectors with respect to the averaged anisotropy axis in Fig. 7.

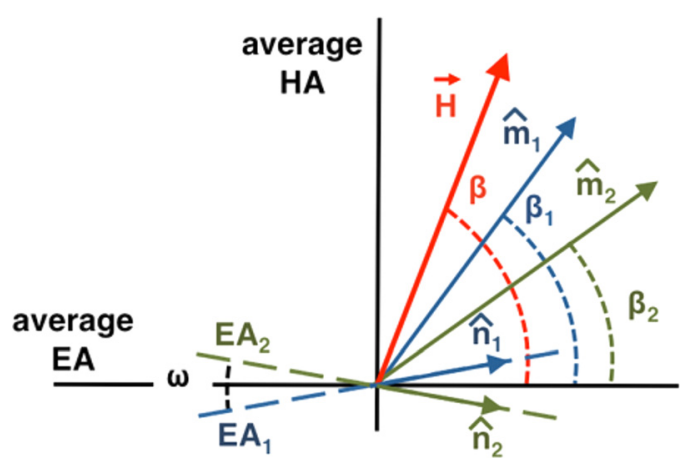

FIG. 7. Schematic of the two grain model geometry with $\omega$ being the misalignment angle between the easy axes of both grains. $\hat{n}_{1}$ and $\hat{n}_{2}$ are the unit vectors along the grains' easy axes. The magnetization vectors are represented by $\hat{m}_{1}$ and $\hat{m}_{2}$, and $\beta_{1}$ and $\beta_{2}$ are the angles between the magnetization vectors and the average easy axis. $\vec{H}$ is the applied field, which is an angle of $\beta$ with respect to the average easy axis.
Using the energy expression given in Eq. (5), we have simulated the magnetization reversal for different $\omega$ and at fixed $J / K$-ratio, starting from the saturated state at high applied field by following the nearest energy minimum upon reducing the applied field. Figure 8 shows a comparison between the experimentally measured $M_{r} / M_{O}$ values and simulated $M_{r} / M_{O}$ values in the vicinity of the nominal HA. Figure 8(a) shows our experimental $M_{r} / M_{O}$ data for the fully epitaxial sample $\left(t_{o x}=0 \mathrm{~nm}\right)$, while Figure 8(c) displays $M_{r} / M_{0}$ simulated values for two perfectly aligned grains $\left(\omega=0^{\circ}\right)$, in which case our two grain model is identical to the conventional SW model. ${ }^{70}$ Figure $8(\mathrm{~b})$ shows our $M_{r} / M_{O}$ measurements for the partially epitaxial sample with $t_{o x}=0.132 \mathrm{~nm}$, while simulated $M_{r} / M_{O}$ values can be seen in Fig. 8(d), for which we made the assumption of a Gaussian distribution of misalignment angles, solving the equilibrium equation for the individual misalignment angles in the distribution and compute a weighted average of the results with a mean value of $\omega=21.5^{\circ}$ and a distribution width of $\Delta \omega=1.2^{\circ}$, as well as a $J / K$ ratio of 0.4 . A clear qualitative agreement is found between the experiments and the simulated curves, and even the quantitative agreement is very good, which is surprising given the simplicity of our twograin model. While the remanent magnetization in the HA vanishes for completely aligned grains [Fig. 8(c)], it increases for misaligned anisotropy angles along the HA direction and in its vicinity [Fig. 8(d)], just as we observed in our experiments.

Figure 9 shows the remanent magnetization along the nominal HA as a function of $t_{o x}$. The experimental data are represented by the cross symbols in the plot and they are compared to the calculated remanent magnetization (solid line) that is predicted by the two grain SW model as a function of the average misalignment angle $\omega$ for $J / K=0.4$ and a

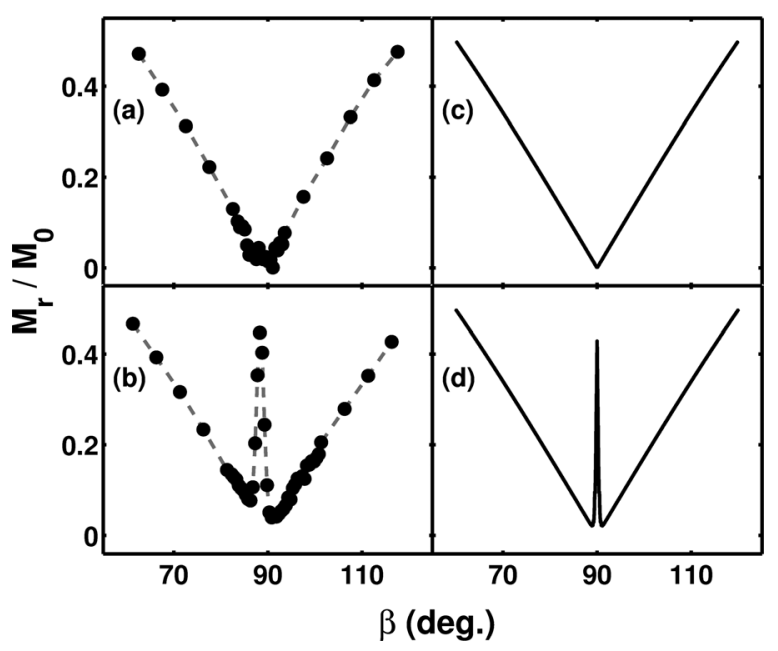

FIG. 8. Angular dependence of the normalized remanent magnetization $M_{r} / M_{0}$ in the vicinity of the hard axis (HA): (a) shows measured $M_{r} / M_{0}$ data for the fully epitaxial sample and (b) the corresponding data for the partially epitaxial sample with $t_{o x}=0.132 \mathrm{~nm}$. (c) and (d) show the calculated $M_{r} / M_{O}$ vs. field angle $(\beta)$ dependence corresponding to the two-grain SW model for aligned anisotropy axes (c) and a Gaussian distribution of misaligned anisotropy axes (d) with a mean misalignment angle of $21.5^{\circ}$ and a standard deviation of $1.2^{\circ}$. For these calculation, we furthermore used the model parameter $J / K=0.4$. 


\section{$\omega$ (deg.)}

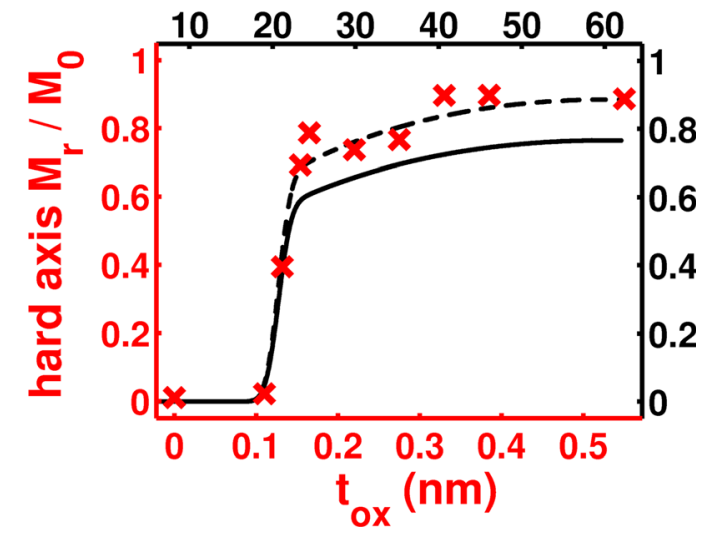

FIG. 9. Sample dependence of the anomalous HA remanence: The crosses show the measured HA remanent magnetization values, normalized to the reference magnetization $\left(M_{0}\right)$, for samples with different $t_{o x}$. The solid line shows the calculated HA remanent magnetization, normalized to the saturation magnetization, as a function of the misalignment angle. Consistent with the plots in Figure 8, the calculations were done by means of the two-grain SW model using a Gaussian distribution of misaligned anisotropy axes with a mean misalignment angle of $\omega$, a standard deviation of $1.2^{\circ}$ and $J / K=0.4$. The dashed line displays the same calculated remanent magnetization curve, but normalized to the reference magnetization, that is achieved at $H=0.3 H_{K}$ for each value of $\omega$, with $H_{K}$ being the single particle anisotropy field.

fixed alignment angle distribution width of $\Delta \omega=1.2^{\circ}$. Both the experiment and the model show a very similar behavior, with the anomalous remanent magnetization appearing abruptly at $t_{o x}=0.132 \mathrm{~nm}$ and $\omega=21.5^{\circ}$ and increasing more slowly for further enhanced disorder levels. This means that our model does not only predict the anomalous behavior, in general, but it also describes its threshold onset behavior. For small enough misalignment angles at any given $J / K$-ratio, there is no anomaly. It takes a non-vanishing critical level of misalignment to be present for the anomaly to occur in the model, in exactly the same way, in which we had to produce a sufficient level of crystallographic misalignment in our uniaxial Co-film samples for the effect to appear.

However, the calculated solid line in Fig. 9 is not an exact match to our experimental data. This may have several reasons. One possibility for this disagreement is that our samples evidently represent a far more complex magnetic system than the two-grain model. Otherwise, one would expect to see a two level magnetization behavior for the anomalous state in Kerr microscopy, which we do not observe [inset of Fig. 6(b)]. Instead one observes a broad distribution of magnetization directions within the domain structure. This aspect is of course a crucial fundamental limitation of our model. The assumption of a Gaussian misalignment angle distribution is a step to overcome this two level magnetization limitation of the model, but it is only a rather simplistic one, because even three particle correlations are already ignored. Second, one would not expect the misalignment angle, $\omega$, and the $\mathrm{SiO}_{2}$ interlayer thickness, $t_{o x}$, to have a linear relationship. In fact, in Fig. 2(c), one can see that amplitude of the $\mathrm{Ag}$ (110) peak does not decrease in a linear fashion as $t_{o x}$ increases. Given that the crystallographic order of the Co-films depends directly on the crystalline structure of the template layers underneath, we can conclude that the crystallographic misalignment angles in the Co films are not expected to be linearly dependent on $t_{o x}$.

However, there is an even simpler experimental reason for the disagreement. The simulated $M_{r}$ data are normalized to the saturation magnetization $M_{s}$, which is of course easily accessible in calculations because it is an input parameter. As discussed earlier in conjunction with Fig. 4, the same is not easily possible for experimental data due to the lack of $M_{s}$ actually being reached in nearly all experimental configurations. Thus, the experimental data are normalized to $M_{O}$ which is in general smaller than $M_{s}$, so that the $M_{r} / M_{0}$-ratio for the experimental data will show larger values than one would see if an $M_{s}$ normalization was done. In order to have a quantitative comparison with the simulations, we have normalized the calculated data to $M_{0}$ values, defined as the magnetization values reached at $H=0.3 H_{K}$ for every set of parameters with $H_{K}$ being the single grain anisotropy field. This field choice seems reasonable, given that the experimental $M_{0}$ was determined at $H=1.8 \mathrm{kOe}$, while $H_{K}$ of Co is known to be 5-6 kOe. The so re-calibrated theoretical curve is shown as a dashed line in Fig. 9, which shows a surprisingly good quantitative agreement with the experimental data.

Given the fact that our two-grain model describes all aspect of the observed experimental HA anomaly and does so with a surprisingly high degree of precision, we can now use this model to analyze the occurrence of this effect. Hereby, it is particularly helpful to analyze the path that the magnetization vectors follow from saturation to remanence for different misalignment angles and field orientations. In the case of completely aligned anisotropies $\left(\omega=0^{\circ}\right)$, both grains behave like a single grain, emulating the SW model, which is characterized by aligned magnetization vectors under all applied field conditions. Increasing the misalignment angle above a threshold or critical angle $\omega_{c}$, however, the system stops to behave as a single grain. This effect is most easily seen when the field is applied exactly along the HA, as shown in Fig. 10(a). Upon decreasing the externally applied field, each of the two-grain magnetizations tries to align with the

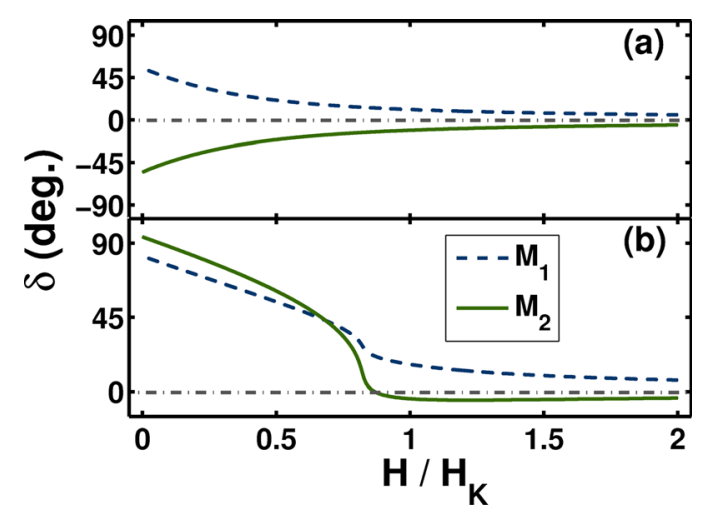

FIG. 10. Field evolution of the individual magnetization angles in the two grain SW model. Here, the magnetization angles $\delta$ are displayed with respect to applied field direction, while the applied field strengths are normalized to the single grain anisotropy field $H_{K}$. The calculations shown here were done for $\omega=21^{\circ}$ and $J / K=0.4$, and for a sample alignment of (a) along the HA $\left(\beta=90^{\circ}\right)$ and (b) $2^{\circ}$ away from the HA $\left(\beta=88^{\circ}\right)$. The dashed line represents the magnetization angle in the first grain and the solid line represents the magnetization angle in the second grain. The grey dasheddotted line represents the applied field direction. 
individual EA direction that is closest to the field orientation. This results in the fact that the two magnetization vectors rotate into opposite directions. At the same time, the exchange coupling tries to align both magnetizations, which has the end result that the magnetization vectors get stuck in a scissor like remanent state with intermediate magnetization orientation angles, for which neither the anisotropy nor the exchange coupling energy are minimized. So, the competition between the exchange coupling energy and anisotropy energy leads to a frustrated state, which also exhibits a high remanent magnetization. Furthermore, this explains the nonuniform remanent magnetization state that we observed by Kerr microscopy, as shown in the inset of Fig. 6(b).

Fig. 10(b) shows the model behavior at an applied field angle of $\beta=88^{\circ}$ for same level of misalignment, i.e., $\omega=21^{\circ}$. It is important to notice that for this level of misalignment, also here the respective closest easy axis directions of the two grains define opposite rotations with respect to the field axis. Thus, as one reduces the magnetic field, a scissor state appears initially at intermediate field values. However, as the field is decreased further, the exchange energy overcomes this initial scissor nucleation and both magnetic moments rotate towards the easy axis that is closer to the field axis. This behavior leads to a nearly uniform magnetization state in remanence, as one can see in Fig. 10(b). For misalignment angles smaller than $\omega_{c}$, the exchange coupling energy dominates the behavior even along the HA and imposes that the magnetic moments rotate together, even though also here a scissor nucleation state is being formed at intermediate field values. In this case, no visible anomaly appears.

The HA anomaly has been also studied by means of BLS spectroscopy, which is a powerful method to study spin dynamics within a ferromagnetic film. Thermally activated spin waves have been analyzed utilizing the inelastic interaction between photons and magnons. In particular, we have studied the effect that the HA anomaly state has onto spinwave spectra. The BLS setup has been equipped with an automated in-plane rotator that allowed for a precise variation of the sample orientation with respect to the externally applied in-plane magnetic field. All measurements have been performed in backward scattering geometry, with the incident light being focused onto the sample under an incident angle of $17.5^{\circ}$ and the scattered light being collected using the same objective. The external field has been applied perpendicular to the incident wave vector.

In order to analyze the effect that the HA anomaly has on the spin-wave spectra, we have measured the dependence of the spin-wave frequencies as a function of the field angle $\beta$ for the $t_{o x}=0.132 \mathrm{~nm}$ sample, for which the anomaly occurs in a very narrow field orientation range. One of the advantages of the BLS technique with respect to the MOKE based technique is that it gives simultaneous information on the average magnetization and its microscopic distribution. Figure 11(a) shows the collected BLS spectra versus $\beta$ in remanence after first applying an external field of 1500 Oe for each angle, while Figure 11(b) shows the exact same type of measurements, but after saturating the sample only once along the EA. The color code contrast indicates the

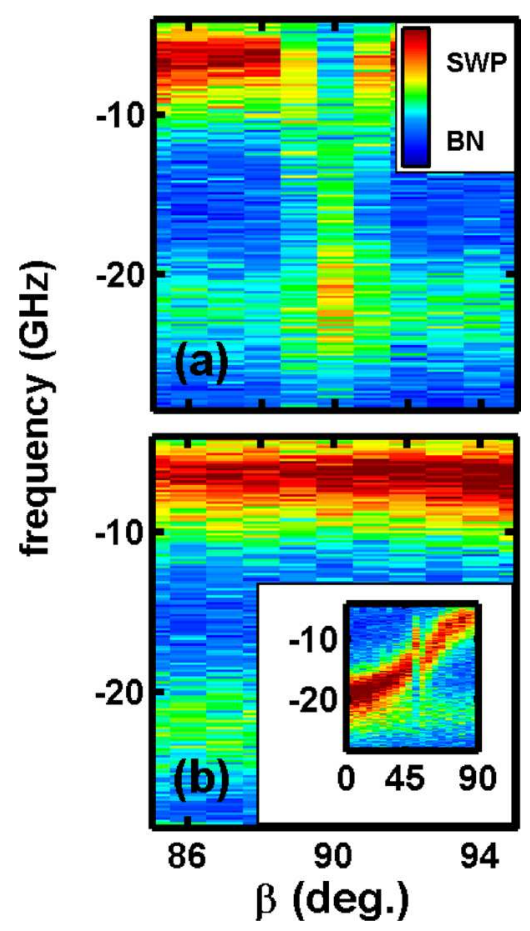

FIG. 11. Brillouin light scattering data of spin wave excitations in the partially epitaxial sample with $t_{o x}=0.132 \mathrm{~nm}$. The color coded plots show the inelastically scattered light intensity as a function of the sample orientation $\beta$ (with respect to the field axis) and the light frequency shift. The color code contrast indicates the spin wave position (SWP) intensity with respect to the background noise $(\mathrm{BN})$. The data were measured (a) after prior saturation at every sample orientation and (b) after prior saturation along the EA, i.e., $\beta=0$. The inset figure in (b) shows the angular dependence at remanence after prior saturation along the EA but for wider angular range of $\beta$.

spin wave position (SWP) intensity with respect to the background noise $(\mathrm{BN})$. In both figures, two spin-wave modes appear, one belonging to the dipolar type spin-wave mode at lower absolute values of frequency and the exchange type perpendicular standing spin wave (PSSW) mode at higher absolute frequency values. ${ }^{76}$ While the frequency of the dipolar dominated spin waves depend, amongst other parameters, on the relative orientation between spin-wave wave vector and magnetization direction, the frequency of the exchange type mode depends mainly on the film thickness. In Fig. 11(a), one can see that due to the changed magnetization orientation along the anomalous HA magnetization reversal, i.e., $\beta=90^{\circ}$, the dipolar type spin wave is shifted towards higher absolute frequency values. ${ }^{76}$ This generally occurring magnetization orientation dependence of dipolar spin wave spectra can be seen clearly in the inset of Fig. 11(b), where the full $90^{\circ}$ dependence is plotted for the same sample after being saturated along the EA. ${ }^{84}$ So, when a field cycle is applied in the vicinity of the HA, but outside the anomalous reversal range, the magnetization aligns with the EA in remanence, which is nearly $90^{\circ}$ away from the applied field direction. However, in the anomalous region, the sample averaged magnetization is forced to stay along the applied field direction, as shown in Fig. 10(a). This causes the upward shift of the dipolar spin-wave frequency in the HA anomaly regime. Furthermore, the data in Fig. 11(b) corroborate that this effect is induced by the field cycling along 
the HA, because no such frequency jump is observed for the same sample orientation in the absence of the applied field sequence. Here, the anomalous state is just never populated.

In addition to the substantial frequency shift, the measurements in Fig. 11(a) show a broadening of the dipolar spin-wave peak in the HA anomalous state. Such a broadening is to be expected, because the anomalous state is characterized by an inhomogeneous sample magnetization, which was observed locally by means of Kerr effect microscopy in Fig. 6(b). Thus, for lateral dimensions larger than the coherence length of the dipolar spin waves, there exists a magnetization orientation distribution, which leads to the observed broadening. The BLS measurements also show that a misalignment of only $\pm 1^{\circ}$ away from the HA already causes an almost complete suppression of this anomalous behavior with a strong reappearance of the $7 \mathrm{GHz}$ low frequency peak. At field orientations of $\pm 2^{\circ}$ from the HA, the usual behavior of uniaxial samples is fully re-established, a result that is also completely consistent with our magnetometry and Kerr microscopy data, i.e., Figs. 8(b) and 6, respectively.

\section{MICROSCOPIC MAGNETIC REVERSAL ANALYSIS}

While much of the previous discussion was focused on the remanent state of magnetization, its angular characteristics and dependence from the anisotropy dispersion, we will now turn our attention to the entire magnetization reversal sequence. It is hereby interesting to recall how the magnetization reversal of perfect single crystal uniaxial films proceeds. Following the discussion of the hysteresis loops shown in Fig. 4, ideal uniaxial systems exhibit two key processes, magnetization rotation and magnetization switching, depending on the magnetic field strength and orientation. For extended in-plane magnetized thin film samples, switching typically occurs by means of a thermally activated local nucleation process of a reverse domain that then expands through the sample via transient domain states after domain wall depinning. ${ }^{17,49,51}$ So, even though the switching segment of the magnetization reversal process away from the HA is associated with non-uniform transient states, i.e., a dynamic reversal sequence, these states are neither stable nor metastable. Thus, on the quasi-static time scale that we analyzed here, ideal ferromagnetic films with uniaxial in-plane anisotropy are characterized by uniform magnetization states only and static, stable, or meta-stable domain states do not exist. Thus, with the introduction of crystallographic misalignment, the biggest qualitative change in magnetization reversal is the possible occurrence of static domain states. We have therefore focused our investigation here on exactly this key aspect of magnetization reversal in films with varying epitaxial alignment: the existence of static reversal domains.

To study the existence of reversal domains, we have performed detailed Kerr effect microscopy studies as a function of the magnetic field angle and strength for our sample series. For this purpose, we utilized an EVICO@Kerr microscopy system in longitudinal geometry. ${ }^{77}$ Our Kerr microscope allows for the simultaneous measurement of local $M(H)$ curves and magnetization state imaging with optical sub-micron lateral resolution and nanoscopic magnetic sensitivity. ${ }^{78}$ Furthermore, the system also allows the study of field orientation dependences by means of a precise sample rotation around the surface normal.

By imaging different magnetization states throughout an entire hysteresis loop cycle, one acquires a vast amount of information. Thus, it is most helpful to extract the most relevant information from these Kerr microscopy data and video sequences to address the relevant scientific question at hand, which in our case is the existence of stable or meta-stable magnetization reversal domains. Thus, in order to have a compact representation of the entire set of magnetization reversal data, we have developed an appropriate representation method. The initial step of this magnetization reversal data extraction and representation method was already reported in a previous paper, ${ }^{50}$ but it will be briefly reviewed here for the purpose of clarity. Figure 12 illustrates how the initial step of our data analysis scheme works. It consists of a pixelby-pixel gray scale analysis for every Kerr microscopy image that is utilized to generate a magnetization projection or orientation histogram to characterize the magnetization state at every field value. Figures 12(a)-12(c) show 3 Kerr microscopy images that were taken during a magnetization reversal sequence for one of our samples. For these images, we have then assembled a gray scale histogram, which can be transformed into a histogram of the magnetization distribution $D(M, H)$, because we have calibrated the grey-scale to
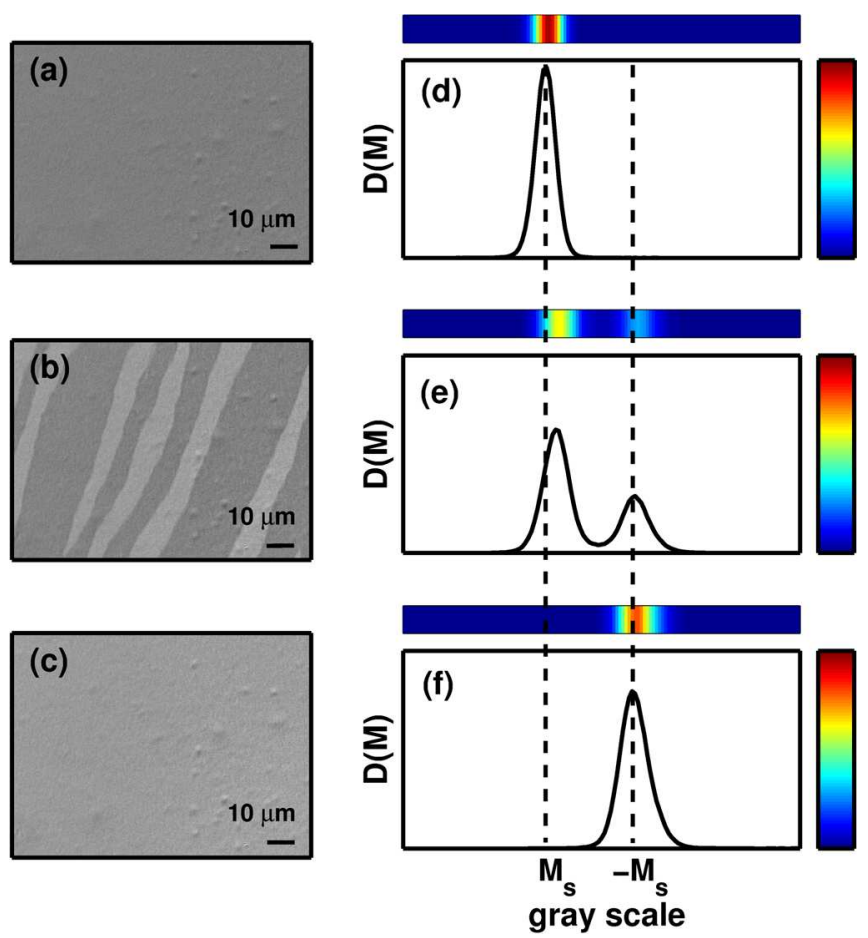

FIG. 12. Histogram representation method of magnetization distributions: Kerr microscopy images are shown for the positive saturation state (a), an intermediate domain state (b), and the negative saturation state (c). The graphs (d)-(f) display the magnetization distribution histograms $D(M)$ that are obtained from images (a)-(c). In addition, the right-hand side of (d)-(f) shows the color code scheme that is being used to represent the $D(M)$ histogram data in a single line each. The corresponding color-coded representations of magnetization distributions are shown above each of the histograms (d)-(f). 
magnetization conversion. Figs. 12(d)-12(f) show the corresponding histograms and furthermore demonstrate how well the histograms are indicative of the existence of domain structures. Fig. 12 also shows how these histograms can be displayed by means of an appropriate color code, so that all crucial information about the magnetization distribution $D(M, H)$ and domain existence in every Kerr image can be displayed in a single color-coded line.

This initial data extraction step, now allows for the display of the field dependent magnetization distribution $D(M, H)$, corresponding to a full video sequence measured via Kerr microscopy, as a single color coded picture. Figure 13(a) shows such a magnetization reversal analysis as a function of the applied field strength for the upper branch of the hysteresis loop (starting from positive saturation and moving towards negative saturation) by using a complete sequence of histograms extracted from the corresponding Kerr microscopy images. In contrast to conventional hysteresis loop measurements, this histogram representation is able to illustrate the entire magnetization distribution as a function of field and not just its average. Correspondingly, one can distinguish in Fig. 13(a) the coherent rotation of the magnetization in between $-30 \mathrm{Oe}$ and $-80 \mathrm{Oe}$ from a non-uniform
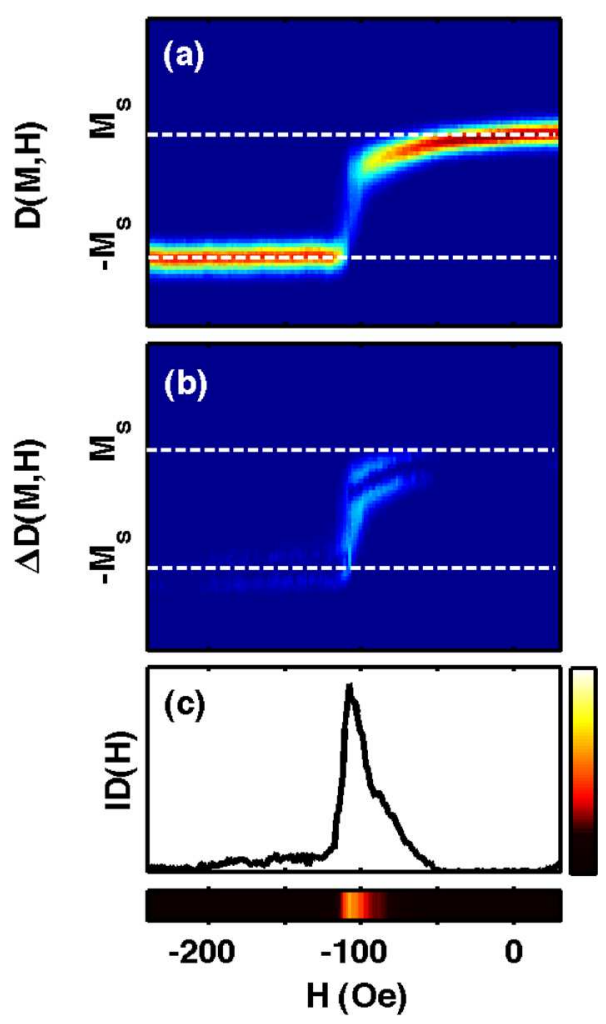

FIG. 13. Schematic of the quantitative domain existence analysis scheme: (a) shows a magnetization reversal sequence for a partially epitaxial sample, which was analyzed by means of the histogram representation method, leading to a $D(M, H)$-map representation of the experimental data. (b) displays the same set of data after removing the uniform magnetization histogram for every field value, leading to the difference map $\Delta D(M, H)$. (c) shows a histogram representation of $I D(H)$, which is derived via to Eq. (6), i.e., by means of a magnetization axis integration of the $\Delta D(M, H)$-map, shown in (b). In addition, the right-hand side of (c) shows the color code scheme that is being used to represent the $I D(H)$ histogram data in a single line. The corresponding color-coded quantitative representation of domain state existence is shown below the histogram (c). state formation starting at about $-80 \mathrm{Oe}$ and extending to approximately $-115 \mathrm{Oe}$. While the coherent rotation or any uniform magnetization reversal process is represented here by a histogram peak shift, domains, or any other nonuniform state formation is shown by a peak splitting or peak broadening. It is therefore possible to formalize the domain state analysis further by subtracting the uniform magnetization state histogram $D_{0}(M, H)$ for every magnetic field value. In this way, one obtains a representation of non-uniform magnetization states only, which is shown in Fig. 13(b). For the numerical analysis, the uniform magnetization histogram was determined at large applied fields and then subtracted for every field value after adjusting for possible peak position shifts. Thus, the magnetization distribution difference $\Delta D(M, H)=\left|D(M, H)-D_{0}(M, H)\right|$ deviates from zero only if non-uniform magnetization states exist. Figure 13(b) clearly displays the occurrence of these non-uniform states during the magnetization reversal in bright color, while the rotation processes that occur exclusively until about $-80 \mathrm{Oe}$ (coming from positive field values) do not show up in this representation.

In order to further formalized the multi-domain state analysis, we have quantified the non-uniform magnetization by integrating the non-uniformity indicator $\Delta D(M, H)$ as a function of $M$, i.e., calculating

$$
I D(H)=\int_{M} \Delta D(M, H) d M,
$$

hereby obtaining the histogram in Fig. 13(c) that quantifies the occurrence of magnetic non-uniformity as a function of the applied field strength. By assigning the color code that is indicated on the right hand side of Fig. 13(c) to this histogram, we are now in a position to obtain a single line representation of the multi-domain state existence range as a function of the externally applied field strength, which is shown at the bottom of Fig. 13(c). Thus, for each thin film sample, a map of all experimental data can be assembled, which identifies the field strength and orientation ranges, in which uniform single domain (SD) states occur or nonuniform MD states are generated during the magnetization reversal process, i.e., exactly the kind of information that characterizes deviations from the simple behavior of perfect uniaxial samples.

Figure 14 shows the multi domain stability ranges during magnetization reversal for 6 of our uniaxial thin film Cosamples with varying degree of anisotropy dispersion. Here, black indicates a single domain state, while other colors reveal the existence of stable or meta-stable multi domain states. While the epitaxial sample in Fig. 14(a) does not show any static multi-domain state as expected, samples with sufficiently thick $\mathrm{SiO}_{2}$ interlayers and correspondingly large anisotropy dispersions show a clearly visible existence range for multi-domain states in Figs. 14(c)-14(f). Moreover, by comparing all the individual figures, it is clear that the multi-domain stability range increases with increased crystallographic misalignment and thus anisotropy dispersion.

In the case of the $t_{o x}=0.11 \mathrm{~nm}$ sample, shown in Fig. 14(b), multi-domain states appears for only one 

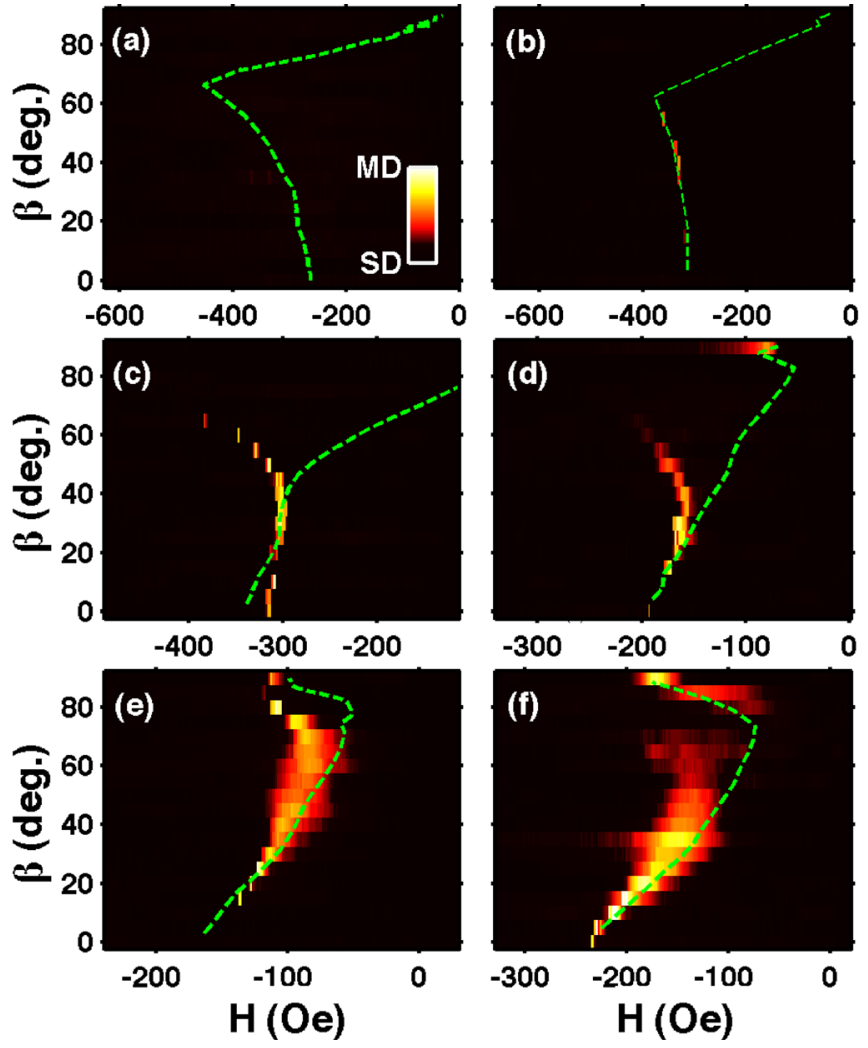

FIG. 14. Domain state existence maps $I D(H, \beta)$ for the magnetization reversal of in-plane uniaxial Co-films with different degree of crystallographic order: (a) fully epitaxial (1010) Co film, and partially epitaxial films with a deposited $\mathrm{SiO}_{2}$ underlayer thickness of (b) $0.11 \mathrm{~nm}$, (c) $0.132 \mathrm{~nm}$, (d) $0.154 \mathrm{~nm}$ (e) $0.165 \mathrm{~nm}$, and (f) $0.275 \mathrm{~nm}$. Also shown as a green dashed line in every plot is the angular dependence of the coercive field, which was extracted from MOKE hysteresis loop measurements. All maps use the same color scheme, which is displayed in (a): black corresponds to $I D=0$, which indicates magnetic uniformity or a SD state, while white corresponds to the maximum value of $I D$ found in any of the data sets, for which the entire field of view of the original Kerr microscopy picture is populated by MD.

measured field strength and only for at few applied field angles. Thus, domain state stability cannot be seen as fully established here. Instead, our domain observation here is a consequence of imaging transient, but rather slowly changing, domain states in the exact moment, at which a domain wall moves through the sample area that we image with our Kerr microscope. This interpretation is consistent with the fact that the appearing pattern of domain wall stability is not reproducible, but changes in between different measurement runs in a statistical manner. ${ }^{53}$

For the samples with $t_{o x}=0.132 \mathrm{~nm}$ and $t_{o x}=0.154 \mathrm{~nm}$, multi-domain structures appear for measurements near the EA and we see that the stability range of these multi-domain states increases as $\beta$ is increased up to around $50^{\circ}$. For even larger values of $\beta$, both samples exhibit a magnetization reversal dominated by uniform magnetization states. So, apparently, it is energetically favorable to reverse the magnetization by coherent rotation rather than domain formation at these high field angles, even in the presence of some crystalline misalignment.

For samples with higher crystallographic misalignment, i.e., $t_{o x}=0.165 \mathrm{~nm}$ and $t_{o x}=0.275 \mathrm{~nm}$, the multi-domain reversal states become even more relevant but also here the stability of non-uniform states decreases for angles near the nominal EA. The HA anomaly is manifested as a multidomain state along or in the vicinity of the HA, which is visible for all samples with $t_{o x} \geq 0.154 \mathrm{~nm}$. Given the $5^{\circ}$ angular $\beta$ resolution of the data sets shown in Fig. 14, the non-uniform anomalous HA state appears for the $t_{o x}=0.154 \mathrm{~nm}$ and $t_{o x}=0.165 \mathrm{~nm}$ samples only when the magnetic field is applied along the HA, while it is already stable at $\beta=85^{\circ}$ for the sample with $t_{o x}=0.275 \mathrm{~nm}$.

On top of all maps in Fig. 14, a green line shows the measured angular dependence of the coercive field $H_{c}$ that we extracted from our MOKE hysteresis loop measurements. The $H_{c}$ curve for the fully epitaxial sample, as well as for sample with $t_{o x}=0.11 \mathrm{~nm}$, shows an initial increase of $H_{c}$ with $\beta$ until around $60^{\circ}$, after which it decreases for even larger magnetic field angles. This type of shape has been previously observed in highly ordered uniaxial samples. ${ }^{17,49,51}$ It indicates a magnetization reversal through a combination of domain nucleation followed by domain wall depinning and rapid propagation for field orientations near the EA. This behavior changes into a coherent rotation reversal for field values $H \cong H_{c}$ upon approaching the HA orientation of the applied magnetic field, simply because the field axis magnetization component reaches its zero value before a switch actually occurs.

For samples with higher anisotropy dispersion, the coercive field vs. applied field angle dependence is very different. We see a decrease of $H_{c}$ with $\beta$ for the samples with $t_{o x} \geq 0.132 \mathrm{~nm}$ and this effect is becoming more pronounced as the disorder increases. It is worthwhile to notice that this qualitative change in behavior happens at the same disorder level, at which stable reversal domains begin to occur. Also, the $H_{c}$ behavior near the HA changes its $\beta$ dependency due to the HA anomaly, which is very visible in Figs. 14(d)-14(f). So, instead of having a vanishing remanent magnetization and coercivity for well aligned systems, the coercivity starts to increase again, as the anomalous domain state get populated for magnetic field orientations near the HA.

For the samples with $t_{o x}=0.132 \mathrm{~nm}$ and $t_{o x}=0.154 \mathrm{~nm}$, $H_{c}$ overlaps with the domain existence range up to applied field angles of around $30^{\circ}$, indicating that the reversal processes at $H_{c}$ are dominated by domain processes. However, as the field angle increases further, the $H_{c}$ line separates from the domain stability range, which is still existent. This simply means that for $\beta>30^{\circ}$, the processes at $H_{c}$ are dominated by magnetization rotation, whereas domain generation occurs only for larger field values near the completion of the magnetization reversal process. For films with higher grain misalignment, such as the samples with $t_{o x}=0.165 \mathrm{~nm}$ and $t_{o x}=0.275 \mathrm{~nm}$, the angular dependence of $H_{c}$ traces the domain existence range closely over most, if not the entire field orientation range. This means that for high disorder levels, domain states control most of the magnetization reversal process, so that also the behavior near the coercive field is dominated by intermediate domain states, almost or entirely independent from the magnetic field orientation, even though these types of films still exhibit very clear uniaxial magnetic anisotropy. 

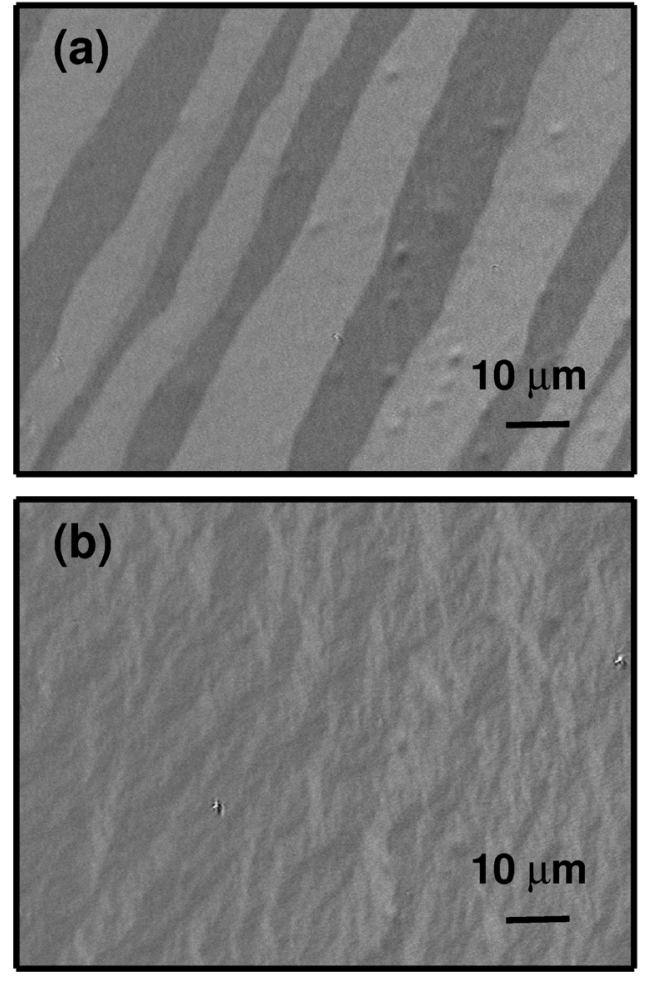

FIG. 15. Domain structures, imaged by Kerr effect microscopy, for samples with $t_{o x}=0.132 \mathrm{~nm}$ (a) and $t_{o x}=0.165 \mathrm{~nm}$ (b) in the vicinity of the coercive field during the magnetization reversal process.

This magnetization reversal difference between lower disorder samples, such as the $t_{o x}=0.132 \mathrm{~nm}$ and $0.154 \mathrm{~nm}$ Co-layers, and higher disorder samples, such as the $t_{o x}=0.165 \mathrm{~nm}$ and $0.275 \mathrm{~nm}$ films, is also reflected in the actual shape of the magnetic domain states. Figure 15 shows domain images taken at an intermediate magnetic field angle for sample $t_{o x}=0.132 \mathrm{~nm}$ (a) and $t_{o x}=0.165 \mathrm{~nm}$ (b). For samples with higher disorder, i.e., Fig. 15(b), the magnetization correlation length is decreased and correspondingly, smaller size domains appear forming ripple like structures, in comparison to the $t_{o x}=0.132 \mathrm{~nm}$ sample, shown in Fig. 15(a), where well defined and bigger domains are generated that, furthermore, exhibit only two possible magnetization values.

\section{CONCLUSIONS}

We have demonstrated, by varying the crystallographic alignment in fully or partially epitaxial Co (1010) films, that their magnetic properties and in particular, the anisotropy dispersion and correspondingly the magnetization reversal processes can be modified in a well defined and reproducible manner. In addition to the detailed non-magnetic characterization of our samples, we have measured their macroscopic and microscopic magnetic properties by means of a self-built MOKE setup and Kerr microscopy. In partially epitaxial films, we have found that the magnetization behavior changes in a non-trivial and unexpected way that cannot be understood by simple orientation averaging. This anomalous behavior arises from the misalignment of the anisotropy axes, which is in competition to the exchange coupling in the
Co-films and causes a disorder induced collective reversal mode that leads to a frustrated magnetic state in remanence. We were able to characterize in detail the features of this anomalous magnetization reversal also in spin wave spectra by BLS technique. Moreover, in order to understand the origin of this behavior, we have devised a model that corroborates all key experimental findings and shows surprisingly good quantitative agreement despite its simplicity.

We also find that the induced crystallographic misalignment and anisotropy dispersion in our films causes the occurrence of non-uniform magnetization reversal, which generally does not occur in highly ordered "perfect" samples. We have studied this phenomenon by means of Kerr microscopy, for which we devised a new data extraction and representation method that allows for a compact and automated analysis of microscopy images. This methodology allowed us to conduct a comprehensive study of domain state stability during magnetization reversal as a function of the magnetic field angle and strength for our entire sample series. By means of this methodology, we observed that domain state stability increases with increasing the disorder level. While for highly ordered samples, no stable domain states occur in the entire field orientation range, samples with larger crystallographic misalignments present stable or meta-stable non-uniform states at virtually all field angles, even though intermediate field angles exhibit the largest field amplitude range of domain stability. Moreover, we have highlighted the relationship between the angular dependence of the coercive field and the existence of reversal domains, because they are not intimately connected in contrast to what may be commonly expected. Actually, we find that not in all cases, the coercive field region is dominated by reversal domains, not even for samples and field orientations, for which domain states occur at some point during the magnetization reversal process.

\section{ACKNOWLEDGMENTS}

We acknowledge funding from the Basque Government for Project No. PI2012-47 and Etortek program (IE11-304) and the Spanish Ministry of Science and Education for Project No. MAT2012-36844. O.I. acknowledges Basque Government for fellowship No. BFI09.284. P.L. acknowledges support from FONDECYT 1120618.

${ }^{1}$ E. J. Torok, J. Appl. Phys. 36, 952 (1965).

${ }^{2}$ F. Schumacher, J. Appl. Phys. 70, 3184 (1991).

${ }^{3}$ I. Tagawa and Y. Nakawa, IEEE Trans. Magn. 27, 4975 (1991).

${ }^{4}$ J. M. Florczak and E. D. Dahlberg, Phys. Rev. B 44, 9338 (1991).

${ }^{5}$ D. C. Jiles, J. Magn. Magn. Mater. 242-245, 116 (2002).

${ }^{6}$ R. J. M. van de Veerdonk, X. Wu, and D. Weller, IEEE Trans. Magn. 38, 2450 (2002).

${ }^{7}$ A. Berger, Y. Xu, B. Lengsfield, Y. Ikeda, and E. E. Fullerton, IEEE Trans. Magn. 41, 3178 (2005).

${ }^{8}$ A. Berger, B. Lengsfield, and Y. Ikeda, J. Appl. Phys. 99, 08 E705 (2006).

${ }^{9}$ A. Romeo, G. Finocchio, M. Carpentieri, L. Torres, G. Consolo, and B. Azzerboni, Physica B 403, 464 (2008).

${ }^{10}$ A. A. Leonov, U. K. Rößler, and A. N. Bogdanov, J. Appl. Phys. 104, 084304 (2008).

${ }^{11}$ O. Hovorka, Y. Liu, K. A. Dahmen, and A. Berger, Appl. Phys. Lett. 95, 192504 (2009).

${ }^{12}$ O. Hovorka, Y. Liu, K. A. Dahmen, and A. Berger, J. Magn. Magn. Mater. 322, 459 (2010). 
${ }^{13}$ W. R. Beam and K. Y. Ahn, J. Appl. Phys. 34, 1561 (1963).

${ }^{14}$ D. Weller, H. Braündle, G. Gorman, C.-J. Lin, and H. Notarys, Appl. Phys. Lett. 61, 2726 (1992)

${ }^{15}$ F. Scheurer, R. Allenspach, P. Xhonneux, and E. Courtens, Phys. Rev. B 48, 9890 (1993).

${ }^{16}$ M. P. Sharrock, J. Appl. Phys. 76, 6413 (1994).

${ }^{17}$ N. P. Suponev, R. M. Grechishkin, M. B. Lyakhova, and Y. E. Pushkar, J. Magn. Magn. Mater. 157/158, 376 (1996).

${ }^{18}$ R. P. Cowburn, S. J. Gray, J. Ferré, J. A. C. Bland, and J. Miltat, J. Appl. Phys. 78, 7210 (1995).

${ }^{19}$ M. Grimsditch, E. E. Fullerton, and R. L. Stamps, Phys. Rev. B 56, 2617 (1997).

${ }^{20}$ A. Khapikov, L. Uspenskaya, J. Ebothe, and S. Vilain, Phys. Rev. B 57, 14990 (1998).

${ }^{21}$ J. S. Jiang, E. E. Fullerton, M. Grimsditch, C. H. Sowers, and S. D. Bader, J. Appl. Phys. 83, 6238 (1998).

${ }^{22}$ Y. Liu, R. A. Thomas, S. S. Malhotra, Z. S. Shan, S. H. Liou, and D. J. Sellmyer, J. Appl. Phys. 83, 6244 (1998).

${ }^{23}$ J. Nogues and I. K. Schuller, J. Magn. Magn. Mater. 192, 203 (1999).

${ }^{24}$ T. Suzuki, N. Honda, and K. Ouchi, J. Appl. Phys. 85, 4301 (1999).

${ }^{25}$ P. Ohresser, J. Shen, J. Barthel, M. Zheng, C. V. Mohan, M. Klaua, and J. Kirschner, Phys. Rev. B 59, 3696 (1999).

${ }^{26}$ R. A. Ristau, K. Barmak, L. H. Lewis, K. R. Coffey, and J. K. Howard, J. Appl. Phys. 86, 4527 (1999).

${ }^{27}$ A. Berger, A. Inomata, J. S. Jiang, J. E. Pearson, and S. D. Bader, Phys. Rev. Lett. 85, 4176 (2000).

${ }^{28}$ X. Liu and G. Zangari, J. Appl. Phys. 90, 5247 (2001).

${ }^{29}$ S. D. Bader, Surf. Sci. 500, 172 (2002).

${ }^{30}$ M. Demand, S. Padovani, M. Hehn, K. Ounadjela, and J. P. Bucher, J. Magn. Magn. Mater. 247, 147 (2002).

${ }^{31}$ W.-T. Lee, S. G. E. te Velthuis, G. P. Felcher, F. Klose, T. Gredig, and E. D. Dahlberg, Phys. Rev. B 65, 224417 (2002).

${ }^{32}$ R. Skomski, J. Phys. Condens. Matter 15, R841 (2003).

${ }^{33}$ N. X. Sun and S. X. Wang, J. Appl. Phys. 93, 6468 (2003).

${ }^{34}$ X. W. Wu, H. Zhou, R. J. M. van de Veerdonk, T. J. Klemmer, and D. Weller, J. Magn. Magn. Mater. 278, 285 (2004).

${ }^{35}$ V. G. Voznyuk, A. Misra, W. D. Doyle, P. B. Visscher, and R. W. Chantrell, J. Appl. Phys. 97, 10N508 (2005).

${ }^{36}$ J. Hamrle, S. Blomeier, O. Gaier, B. Hillebrands, R. Schaüfer, and M. Jourdan, J. Appl. Phys. 100, 103904 (2006).

${ }^{37}$ A. Aktag, S. Michalski, L. Yue, R. D. Kirby, and S.-H. Liou, J. Appl. Phys. 99, 093901 (2006).

${ }^{38}$ G. S. Chang, A. Moewes, S. H. Kim, J. Lee, K. Jeong, C. N. Whang, D. H. Kim, and S.-C. Shin, Appl. Phys. Lett. 88, 092504 (2006).

${ }^{39}$ M. Kisielewski, A. Maziewski, and V. Zablotskii, J. Magn. Magn. Mater. 316, 277 (2007).

${ }^{40}$ M. F. Chioncel, H. S. Nagaraja, F. Rossignol, and P. W. Haycock, J. Magn. Magn. Mater. 313, 135 (2007).

${ }^{41}$ O. Hellwig, A. Berger, J. B. Kortright, and E. E. Fullerton, J. Magn. Magn. Mater. 319, 13 (2007).

${ }^{42}$ J. Cao, N. T. Nam, S. Inoue, H. Y. Y. Ko, N. N. Phuoc, and T. Suzuki, J. Appl. Phys. 103, 07F501 (2008).

${ }^{43}$ C. A. F. Vaz, J. A. C. Bland, and G. Lauhoff, Rep. Prog. Phys. 71, 056501 (2008).

${ }^{44}$ O. Gaier, J. Hamrle, S. J. Hermsdoerfer, H. Schultheiß, B. Hillebrands, Y. Sakuraba, M. Oogane, and Y. Ando, J. Appl. Phys. 103, 103910 (2008).

${ }^{45}$ C. Mathieu, V. R. Inturi, and M. J. Hadley, IEEE Trans. Magn. 44, 431 (2008).

${ }^{46}$ A. Berger, N. Supper, Y. Ikeda, B. Lengsfield, A. Moser, and E. E. Fullerton, Appl. Phys. Lett. 93, 122502 (2008).

${ }^{47}$ H. Stillrich, C. Menk, R. Frömter, and H. P. Oepen, J. Appl. Phys. 105, 07C308 (2009).

${ }^{48}$ J. Brandenburg, R. Hühne, L. Schultz, and V. Neu, Phys. Rev. B 79, 054429 (2009).

${ }^{49}$ X. D. Liu, Z. Xu, R. X. Gao, Z. F. Chen, T. S. Lai, J. Du, and S. M. Zhou, J. Appl. Phys. 106, 053907 (2009).

${ }^{50}$ O. Idigoras, P. Vavassori, J. M. Porro, and A. Berger, J. Magn. Magn. Mater. 322, L57 (2010).

${ }^{51}$ M. Mathews, E. P. Houwman, H. Boschker, G. Rijnders, and D. H. A. Blank, J. Appl. Phys. 107, 013904 (2010).

${ }^{52}$ S. Trudel, O. Gaier, J. Hamrle, and B. Hillebrands, J. Phys. D. Appl. Phys. 43, 193001 (2010).
${ }^{53}$ J. A. Arregi, O. Idigoras, P. Vavassori, and A. Berger, Appl. Phys. Lett. 100, 262403 (2012)

${ }^{54}$ A. Berger, Physica B: Condens. Matter 407, 1322 (2012).

${ }^{55}$ L. Sun, Y. Wang, M. Yang, Z. Huang, Y. Zhai, Y. Xu, J. Du, and H. Zhai, J. Appl. Phys. 111, 07A328 (2012).

${ }^{56}$ A. K. Suszka, O. Idigoras, E. Nikulina, A. Chuvilin, and A. Berger, Phys. Rev. Lett. 109, 177205 (2012)

${ }^{57}$ V. Inturi, H. Yin, M. Kief, M. Hadley, and C. Mathieu, IEEE Trans. Magn. 48, 1718 (2012).

${ }^{58}$ M. N. Baibich, J. M. Broto, A. Fert, F. Nguyen Van Dau, F. Petroff, P. Eitenne, G. Creuzet, A. Friederich, and J. Chazelas, Phys. Rev. Lett. 61, 2472 (1988).

${ }^{59}$ G. Binash, P. Grünberg, F. Saurenbach, and W. Zinn, Phys. Rev. B 39, 4828 (1989).

${ }^{60}$ A. Fert, Thin Solid Films 517, 2 (2008).

${ }^{61}$ D. S. Schmool, Nanosci. Nanotechnol. Lett. 3, 515 (2011).

${ }^{62} \mathrm{G}$. Bertotti, Hysteresis in Magnetism, for Physicists, Materials Scientists, and Engineers (Academic Press, Inc., San Diego, California, 1998).

${ }^{63} \mathrm{~J}$. Ferré, in Spin Dynamics in Confined Magnetic Structures I, Topics of Applied Physics, Vol. 83, edited by B. Hillebrands and K. Ouenadjela (Springer, Berlin, Heidelberg, 2002), pp. 127-168.

${ }^{64}$ J. F. Herbst, J. J. Croat, F. E. Pinkerton, and W. B. Yelon, Phys. Rev. B 29, 4176 (1984)

${ }^{65}$ D. Weller, J. Stohr, R. Nakajima, A. Carl, M. G. Samant, C. Chappert, R. Megy, P. Beauvillain, P. Veillet, and G. A. Held, Phys. Rev. Lett. 75, 3752 (1995).

${ }^{66}$ J. P. Liu, C. P. Luo, Y. Liu, and D. J. Sellmyer, Appl. Phys. Lett. 72, 483 (1998)

${ }^{67}$ R. Wood, IEEE Trans. Magn. 36, 36 (2000).

${ }^{68}$ V. F. Puntes, P. Gorostiza, D. M. Aruguete, N. G. Bastus, and A. P. Alivisatos, Nature Mater. 3, 263 (2004).

${ }^{69}$ S. N. Piramanayagam and K. Srinivasan, J. Magn. Magn. Mater. 321, 485 (2009).

${ }^{70}$ E. C. Stoner and E. P. Wohlfarth, Philos. Trans. R. Soc. London, Ser. A 240, 599 (1948).

${ }^{71}$ W. Yang, D. N. Lambeth, and D. E. Laughlin, J. Appl. Phys. 85, 4723 (1999).

${ }^{72}$ L. Vegard, Philos. Mag. 31, 83 (1916).

${ }^{73}$ K. E. Johnson, M. Mirzamaani, and M. F. Doerner, IEEE Trans. Magn. 31, 2721 (1995).

${ }^{74}$ M. F. Toney, E. E. Marinero, and J. A. Hedstrom, J. Appl. Phys. 99, 033907 (2006).

${ }^{75}$ O. Idigoras, A. K. Suszka, P. Vavassori, P. Landeros, J. M. Porro, and A. Berger, Phys. Rev. B 84, 132403 (2011).

${ }^{76}$ B. Hillebrands, in Light Scattering Solids VII, edited by M. Cardona and G. Güntherodt (Springer-Verlag, Heidelberg, Germany, 1999).

${ }^{77}$ A. Neudert, J. Mccord, D. Chumakov, R. Schäfer, and L. Schultz, Phys. Rev. B 71, 134405 (2005)

${ }^{78}$ E. Nikulina, O. Idigoras, P. Vavassori, A. Chuvilin, and A. Berger, Appl. Phys. Lett. 100, 142401 (2012).

${ }^{79}$ O. Idigoras, U. Palomares, A. K. Suszka, L. Fallarino, and A. Berger, Appl. Phys. Lett. 103, 102410 (2013).

${ }^{80}$ The main reason to use $M_{O}$ instead of the saturation magnetization value $\left(M_{s}\right)$ for the normalization is the fact that $M_{s}$ is a somewhat elusive experimental quantity, because only along the easy or the hard axis one can actually achieve $M=M_{s}{ }^{79}$ For all other directions, this is not possible and one would have to extrapolate the data to estimate $M_{s}$ in these directions. So, given that the specific value of $M_{S}$ does not contribute any substantial insight to the subject of this study, we have preferred to use $M_{O}$ as a reliable experimental magnetization value for the data normalization.

${ }^{81}$ Each magnetic state image displays the difference between the positive remanent magnetization state and the negative remanent magnetization state in order to increase the contrast.

${ }^{82}$ Even though it would be more precise to devise a model with a more complex angular distribution, ${ }^{32}$ it is not necessary in order to understand the HA anomaly, since the two grain SW model is able to explain all key characteristics of the experimentally observed behavior.

${ }^{83}$ In Eqs. (4) and (5), $E$ defines the volume integrated energy over the two particles and thus all the coefficients including the field are given in energy units.

${ }^{84}$ Note that the abrupt frequency shift around $50^{\circ}$ observed in the inset of Fig. 11(b) arose from a dust particle that was illuminated while rotating. It is not reproducible when changing the illumination area. 